\title{
"Cuts in Action": A High-Density EEG Study Investigating the Neural Correlates of Different Editing Techniques in Film
}

\author{
Katrin S. Heimann, ${ }^{\text {a }}$ Sebo Uithol, ${ }^{\text {b,c }}$ Marta Calbi, ${ }^{\text {d }}$ Maria A. Umiltà, ${ }^{\text {d }}$ \\ Michele Guerra, ${ }^{\mathrm{e}}$ Vittorio Gallese ${ }^{\mathrm{d}, \mathrm{f}}$

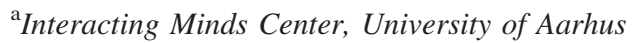 \\ ${ }^{\mathrm{b}}$ Charité Universitätsmedizin Berlin, Bernstein Center for Computational Neuroscience \\ ${ }^{\mathrm{c}}$ Donders Institute for Brain, Cognition and Behaviour, Radboud University Nijmegen \\ ${ }^{\mathrm{d}}$ Department of Neuroscience, University of Parma, Unit of Physiology \\ ${ }^{\mathrm{e}}$ Department of Arts and Literature, History and Social Studies, University of Parma \\ ${ }_{\mathrm{f}}^{\mathrm{f}}$ Institute of Philosophy, School of Advanced Study, University of London
}

Received 22 June 2015; received in revised form 25 April 2016; accepted 10 June 2016

\begin{abstract}
In spite of their striking differences with real-life perception, films are perceived and understood without effort. Cognitive film theory attributes this to the system of continuity editing, a system of editing guidelines outlining the effect of different cuts and edits on spectators. A major principle in this framework is the $180^{\circ}$ rule, a rule recommendation that, to avoid spectators' attention to the editing, two edited shots of the same event or action should not be filmed from angles differing in a way that expectations of spatial continuity are strongly violated. In the present study, we used high-density EEG to explore the neural underpinnings of this rule. In particular, our analysis shows that cuts and edits in general elicit early ERP component indicating the registration of syntactic violations as known from language, music, and action processing. However, continuity edits and cuts-across the line differ from each other regarding later components likely to be indicating the differences in spatial remapping as well as in the degree of conscious awareness of one's own perception. Interestingly, a time-frequency analysis of the occipital alpha rhythm did not support the hypothesis that such differences in processing routes are mainly linked to visual attention. On the contrary, our study found specific modulations of the central mu rhythm ERD as an indicator of sensorimotor activity, suggesting that sensorimotor networks might play an important role. We think that these findings shed new light on current discussions about the role of attention and embodied perception in film perception and should be considered when explaining spectators' different experience of different kinds of cuts.
\end{abstract}

Correspondence should be sent to Katrin S. Heimann, Interacting Minds Center, University of Aarhus, building 1483, Jens Chr. Skous Vej 3, 8000 Aarhus C, Denmark. E-mail: katrinheimann@gmail.com 
Keywords: EEG; Mu-rhythm; Action-perception links; Cognitive film science; Continuity editing; Cuts-across-the-line; Embodied cognition; Attentional theory of continuity editing

\section{Introduction}

Film is omnipresent in today's world, produced and consumed for purposes as diverse as information, advertisement, amusement, and art (Casetti, 2015). One may hypothesize that this popularity is due to the fact that film records life events; therefore, it best simulates perception in the real world and thus strongly involves the perceiver in its depiction. This assumption, however, ignores the fact that only in rare cases a movie is just raw footage - an unaltered continuous shot of one ongoing scene. Rather, what we commonly refer to with the term "film" is a stream of edited moving images consisting of hundreds and thousands of individual camera shots patched together. As such, film differs considerably from what we are used to perceiving "off screen."

It has been suggested that the ease with which spectators still follow a film is crucially dependent on its skilled production, developed to hide the medium's deviating nature by adapting it to the perceiver's perceptual and cognitive capacities and needs. A major role here has been attributed to an editing tradition, commonly referred to as "continuity editing" (Bordwell, 1985; Bordwell \& Thompson, 2006; Cutting, 2005). This editing technique is based on a number of practical instructions for film postprocessing, precisely describing which kind of shots (regarding objects in focus, length and on-/offset of shot, as well as angles of camera position, etc.) should be edited together to best guarantee the still undisturbed perception of a movie. One of the most prominent guidelines among such instructions is the " $180^{\circ}$ rule." According to this rule, the initial shot of a scene draws an imaginary line, called the axis or centerline, which divides the action space in two halves: the first one is where the camera is located (as being placed within a circle orthogonally focusing on the $\left(180^{\circ}\right)$ division line, with the action taking place at the center), while the second one is on the other side of that line (see Fig. 1). This setup then creates a "stage" situation, allowing the camera position to be varied between shots, as long as the centerline is not crossed.

In an attempt to explain this rule in cognitive terms, it has been suggested that the edits containing small camera displacements are still perceptually close enough to normal vision which also includes short interruptions of sight due to the suppression of vision during saccades and blinks. In combination with other bodily movements, such as head or body turns or steps, these eye movements would lead to almost jump-like changes of perspective and therefore of perceived object positions, if they were not automatically overcome by remapping processes based on prior sensorimotor feedback. It was thus hypothesized that the continuity edits are processed with the help of the same remapping processes, while cuts across the centerline create changes too large to be accommodated for in this way. The detection of such changes might lead to heightened attention and different further processing, making it more likely that an edit reaches our conscious awareness and disturbs the perception of the plot (see, for example, Magliano \& Zacks, 2011). 


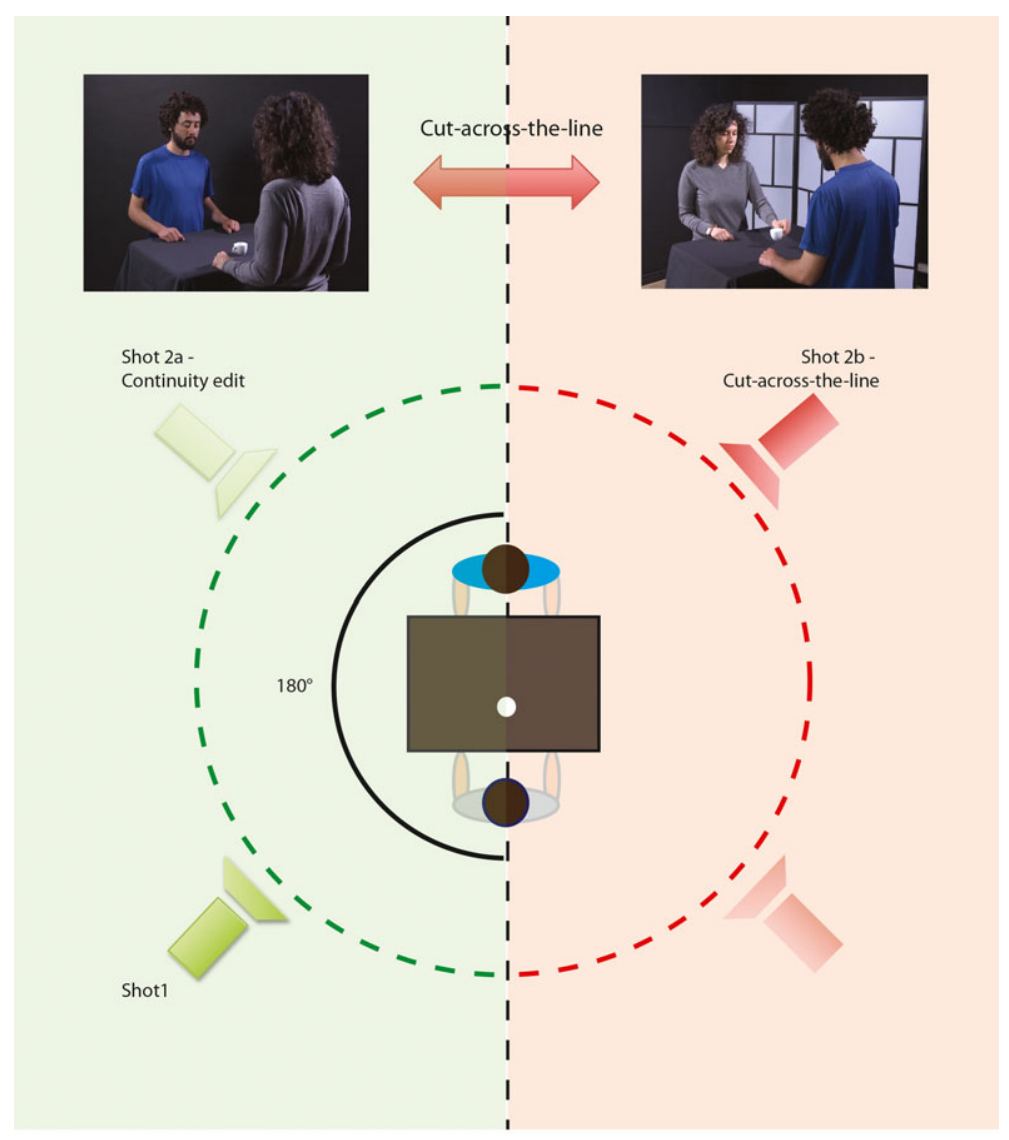

Fig. 1. An illustration of camera displacements between shots complying with or violating the $180^{\circ}$ rule when edited together.

More generally, it was proposed that the better the image stream itself fulfills our expectations of what comes next-based on our usual way of perceiving off-screen - the less the salient changes are, and therefore the deeper our immersion in the film (see also Cutting, 2005; Smith, 2006, 2012).

This, however, is a problematic suggestion, given the fact that movies have not only surrounded us for more than a century, but can also be described as steadily evolving toward a more radical, as opposed to conservative, use of narrative devices (see, for example, the steady decrease of shot length across a century of filmmaking as demonstrated in Salt [2006]). Following these lines, it could be argued that spectators adapt to the medium. Additionally, it has been suggested that spectators are much more driven by the content of the film than by its structure (see Murch, 1992).

Only a limited number of empirical studies investigated the effect of movie cuts on spectators. Lang, Geiger, Strickwerda, and Sumner (1993) found that cuts, in general, elicit typical physiological and behavioral signs of an orienting response, such as decreased 
heart rate, heightened skin conductance, and modulated memory performance for content presented after the cut. These responses are traditionally associated with heightened attention, as well as arousal and cognitive load. This fits to prior findings of Reeves et al. (1985), who observed that cuts are followed by a decrease in central and occipital alpha oscillation, which they interpreted as indicating heightened visual attention. Also, recent events related potential (ERP) studies of Francuz and Zabielska-Mendyk (2013) and Matran-Fernandez and Poli (2015) support this claim by showing that cuts are followed by ERP components that are typical for an orienting response (P300 and slow cortical potential $[\mathrm{SCP}])$.

Research trying to differentiate physiological responses across different kind of cuts or edits is even more rare. A notable exception is the study by Francuz and ZabielskaMendyk (2013), who compared unrelated cuts, that is, cuts between two different, not obviously directly connected scenes, and related cuts, that is, edits showing the same scene filmed from different camera positions. They found that for unrelated cuts, the SCP was of higher amplitude, indicating higher attention, arousal, and cognitive load elicited by this type of stimuli. Furthermore, Magliano and Zacks (2011) investigated blood oxygen leveldependent responses during the observation of (a) "continuity edits," that is, edited shots showing the same ongoing scene but recorded from different camera positions, while the centerline is not crossed; and (b) non-continuity edits including "cuts-across-the-line," that is, edited shots showing the same ongoing scene but recorded from different camera positions, while the centerline is crossed. For continuity edits, they found transient increased activation in cortical areas including the inferotemporal cortex, posterior superior temporal sulcus, and precuneus, and reduced activation in precentral sulcus, as well as in other premotor areas. They interpreted the first activations as being due to stimulus-driven processing serving the remapping of visual features across the editing boundaries. As a result, the cut is not consciously perceived and the event is perceived as unbroken. Reduction of activation in premotor areas was interpreted as being due to attention-driven downregulation. On the other hand, for cuts-across-the-line, fMRI data revealed a transient bilateral increase of activation in the parahippocampal cortex and a decrease of activation in early visual cortices, which they interpreted as reflecting downregulation of activity in regions of visual remapping. As a result, such cuts are not completely masked and disturb continuous perception, possibly leading to their conscious detection. Indeed, previous research by d'Ydewalle and Vanderbeeken (1990) and Schröder (1990) has indicated that cuts that violate the $180^{\circ}$ rule are easier to detect than cuts complying with this rule (Schröder, 1990; d'Ydewalle \& Vanderbeeken, 1990). Magliano and Zacks proposed that the downregulation of visual remapping is likely to be attention-driven, depending on the salient unexpected visual changes of the cuts-across-the-line; however, their fMRI findings do not provide support this claim.

Additionally, there is evidence that attention might not be the crucial factor causing the different processing routes implied by these findings. When comparing unrelated cuts with related cuts, Lang et al. (1993) and Geiger and Reeves (1993) did find behavioral differences in the response, suggesting different processing routes. However, Lang et al. (1993) could not find a modulation of physiological measures (heart rate and skin 
conductance) across conditions, as expected from attentional differences due to varying salience of the stimuli. Furthermore, Germeys and D'Ydewalle (2007) showed that eye movements after cuts-across-the-line do not show signs of orienting responses and related attentional differences, compared to continuity edits.

In our study, we therefore decided not to focus on markers of attention, but instead on the involvement of other mechanisms in the processing of edits. Specifically, we were interested in using ERP responses to investigate the nature and timing of violation detection and processing that according to Magliano and Zacks (2011) followed an edit. Furthermore, we used time-frequency analyses to investigate a possible involvement of sensorimotor networks usually activated during action observation.

There are reasons to assume that action processing mechanisms play a role in perception of moving images as well. First of all, definitions of continuity editing explicitly state the goal of continuity editing to be "the perception of continuous action from discontinuous sensory information" (Smith, 2006; p. 168). It is thus not the continuity of images that is marked as the crucial condition, but of the events and especially actions represented by them. Fittingly, Schwan and Ildirar (2010), investigating the reactions of media-unexperienced individuals to film, have shown that not all edited moving images are readily understood without prior exposure to them. Participants showed severe problems in giving a standard interpretation of the plots when the flow of events and actions, as known from real life, was strongly interrupted, reversed, or destroyed by the editing. Second, Magliano and Zacks (2011), in the experiment described above, did not only find differences between continuity edits and cuts-across-the-line in the premotor cortex - a region typically involved in action planning. When additionally comparing unrelated cuts with related cuts, they also showed a reduction of activation for unrelated cuts in the lateral parietal cortex, a region associated with the representation of action goals (Hamilton \& Grafton, 2009). Lastly, Heimann, Umilta, Guerra, and Gallese (2014) showed by means of high-density Electroencephalography (EEG) a modulation of motor cortex activity during action observation depending on the camera movements employed for filming the action scene. Specifically, their results revealed that the observation of movieclips recorded with a steadicam, a filming technique that preserves part of the walking movements of the cameraman, made the moving image appear as "most naturally representing the scene" and "giving the strongest impression of the spectator himself approaching the scene." Furthermore, the observation of such clips correlated with stronger desynchronization of the rolandic mu-rhythm (a marker of motor cortex activation, see Muthukumaraswamy and Johnson [2004]) than during the observation of clips recorded with camera movements that produced images of more artificial character (zoom and dolly).

In the experiment reported here, we therefore investigated spectators' responses to different cuts using high-density EEG recording and focusing on (a) markers for semantic and syntactic processing in general; and (b) action observation specifically. We hypothesized that in clips showing actions, cuts-across-the-line, due to their sudden reversal of left and right and direction of the action (an effect unfamiliar to us from perception off screen) will cause:

1. The registration of a syntactic (not semantic) violation; 
2. A sensorimotor disturbance that could play a role in spectators' different experiences of such edit.

To test these hypotheses, we analyzed ERPs focusing on components associated with the detection and reanalysis of semantic and syntactic violations (in language, music, and action sequences, see below), as well as event related desynchronization (ERD) of the central mu-rhythm, as this is a known marker of motor cortex activity during action observation. Lastly, as a control, we analyzed also posterior alpha-rhythm as this is interpreted as a marker for visual attention during the first seconds after montage.

Previous ERP studies described a number of component complexes associated with the detection and the reanalysis of specific violations in language, music, and action observation, quite similar across domains (regarding language, see Kutas \& Hillyard, 1980; Kutas \& Federmeier, 2000; Lau, Phillips, \& Poeppel, 2008; regarding music, see Steinbeis \& Koelsch, 2008; Koelsch, 2011). Most interesting for our study, Maffongelli et al. (2015) investigated perception of actions that were suggested by static images (comic-like). They reported that the introduction of semantic violations, by means of the use of an unexpected element in an action such as brushing your hair with a toothbrush, elicited a frontally distributed negative deflection around $400 \mathrm{~ms}$ after stimulus-onset. In contrast to this, structure or syntactic violations, that is, the reversal of event order in such simple actions, elicited an earlier and specifically left anterior negativity (ELAN). Furthermore, when task relevant, these early anterior left negativities were followed by a late positivity in the same region, probably indicating the reanalysis of the stimulus ("violation repair," see Maffongelli et al., 2015). We focused on these components with the following questions: (a) do edits based on a change in camera position in general elicit responses indicating the detection and processing of semantic or syntactic violations; and (b) are such responses modulated by the circumstance of the edit either conforming or violating the $180^{\circ}$ rule? More precisely, do continuity edits and cuts-across-the-line regarding have different effects on the early components associated with stimulus detection and or later ones linked to updating mechanisms following the detection of a violation of an observed action?

In addition, we investigated whether the ERD of the central mu-rhythm would be differentially modulated by the two different types of cuts. The central mu-rhythm has previously been described to be attenuated during the execution as well as during observation of goal related and has therefore been related to the activity of the mirror neuron mechanism in humans, (Perry, Troje, \& Bentin, 2010; Pfurtscheller \& Lopes da Silva, 1999). The mirror neuron mechanism is suggested to instantiate an action perception link involved in action and motor intention understanding (Gallese, 2007; Gallese \& Sinigaglia, 2011; Uithol, van Rooij, Bekkering, \& Haselager, 2011). Furthermore, the activation of the mirror mechanism has been reported to be modulated by perceivers' expertise with the observed actions. Specifically, motor familiarity with the actions observed has been associated with stronger activation of the cortical motor system (Calvo-Merino, Glaser, Grèzes, Passingham, \& Haggard, 2005; Calvo-Merino, Grèzes, Glaser, Passingham, \& Haggard, 2006; Orgs, Dombrowski, Heil, \& Jansen-Osman, 2008). 
We therefore expect to find stronger ERD after continuity edits, as they are perceptually closer to everyday action perception. An analysis of occipital alpha ERD served to control for effects due to visual attention.

\section{Materials and experimental setup}

\subsection{Participants}

Twenty healthy volunteers (10 male, 10 female, $\left.M_{\text {age }} 24.8[S D=2.31]\right)$, all right handed as assessed by (an Italian adaption of) the Edinburgh Handedness Inventory (Oldfield, 1971), participated in the experiment. Participants were recruited by public announcement and were reimbursed with 25 Euros. Before the experiment, they received experimental instructions and gave written informed consent. After the experiment, each participant was debriefed. The study was approved by the local Ethics Committee.

\subsection{Stimuli}

Video clips were recorded and edited in a professional film studio, enabling us to film the same scene under highly controlled conditions. Video clips were of $5 \mathrm{~s}$ length each. Each consisted of two single shots edited together, showing the same scene from different camera positions. In the first shot of $2 \mathrm{~s}$ length, two agents (one male, one female) stand in front of a table, at the center of which a small object (either a salt shaker or an espresso cup) is placed. The actors first look at each other before one of them directs his or her gaze to the object, the other following the gaze of the first actor. In the second shot of $3 \mathrm{~s}$ length, the actor who was the first looking down at the object in shot number one then grasps the object, picks it up, and places it right in front of the other actor. The variable specifically manipulated for the experiment was the angle by which the camera position varied from the first to the second shot edited together. In total, four cameras were used, all at the same distance and height from the center of the scene, $45^{\circ}$ from the centerline. The angle of visual field was approximately $45^{\circ}$ for the diagonal (thereby approaching the [central part of the] human visual field). In $50 \%$ of the clips, the two different cameras used for the two edited shots were placed on one side of the axis; thus, the edit did not violate the $180^{\circ}$ rule. In the other $50 \%$, the camera used for shot one was placed on one side and the camera used for shot two on the other side of the axis, thereby violating the $180^{\circ}$ rule. The total number of clips used was 16 (eight for each condition, counterbalanced for gender of grasping actor, object and first camera position). Fig. 2 shows three still frames demonstrating the effect of the different camera displacements.

\subsection{Experimental procedure, recording, and analysis}

For the reader's convenience, the following paragraphs only describe the fundamental features of the study design and analysis. For figures, please see the Appendix. 

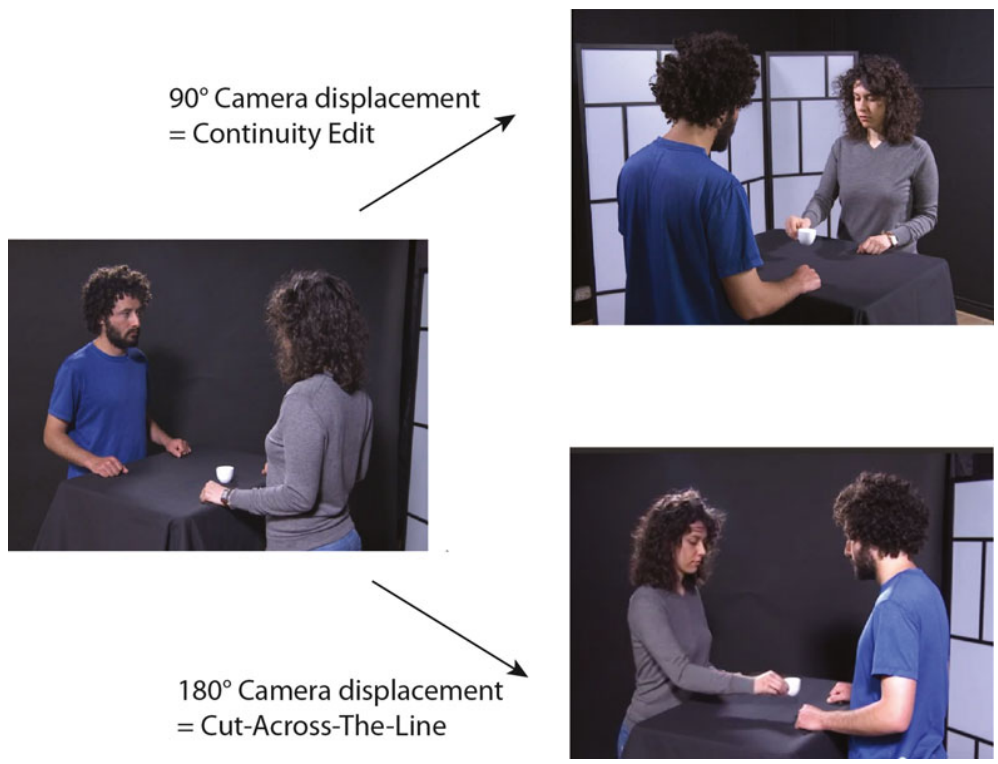

Fig. 2. An illustration of the two different applied edits, one involving a $90^{\circ}$ camera displacement (continuity edit), and the other one a $180^{\circ}$ camera displacement (cut-across-the-line) across the edit.

The experiment consisted of two different parts comprising: 1) a 60-min EEG recording session (including breaks); 2) a 10-min rating task.

\subsubsection{Experimental design and EEG recording details}

Participants were seated in an isolated EEG lab in front of a computer screen placed on a table at a distance of $50 \mathrm{~cm}$. EEG data were acquired by a 128-channel Sensor Net (Electrical Geodesics, Inc. Eugene, USA) and recorded within standard EGI package Net Station 4.3.1. Stimuli were presented with E-Prime 2.0 and at the beginning of each trial, all event markers were sent to the recording software Net Station. EEG was sampled at $500 \mathrm{~Hz}$, and band-pass filtered at $0.1-100 \mathrm{~Hz}$, electrode impedance was kept less than $50 \mathrm{~K} \Omega$ (controlled after each block). The raw EEG data were recorded with the vertex $(\mathrm{Cz})$ as the online reference and re-referenced off-line to the common average (Muthukumaraswamy, Johnson, \& McNair, 2004).

During the EEG recording, a video camera recorded participants for off-line analysis of their movements; if participants moved during the observation or baseline periods, the trial was excluded from further analysis. Participants were instructed to blink as little as possible during trials to reduce eye movement-related artifacts.

EEG was recorded during three blocks of about $15 \mathrm{~min}$ (53-54 trials each, 80 trials in total per condition, thus repeating each clip 10 times). Each trial began with a fixation cross (of random duration between 500 and 1,000 ms), followed by one of the video stimuli (of $5 \mathrm{~s}$ length each, presented in pseudo-random order). In $50 \%$ of the trials, after stimulus presentation, first a green screen (2 s), then a gray screen (ITI, $3 \mathrm{~s}$ ) were 


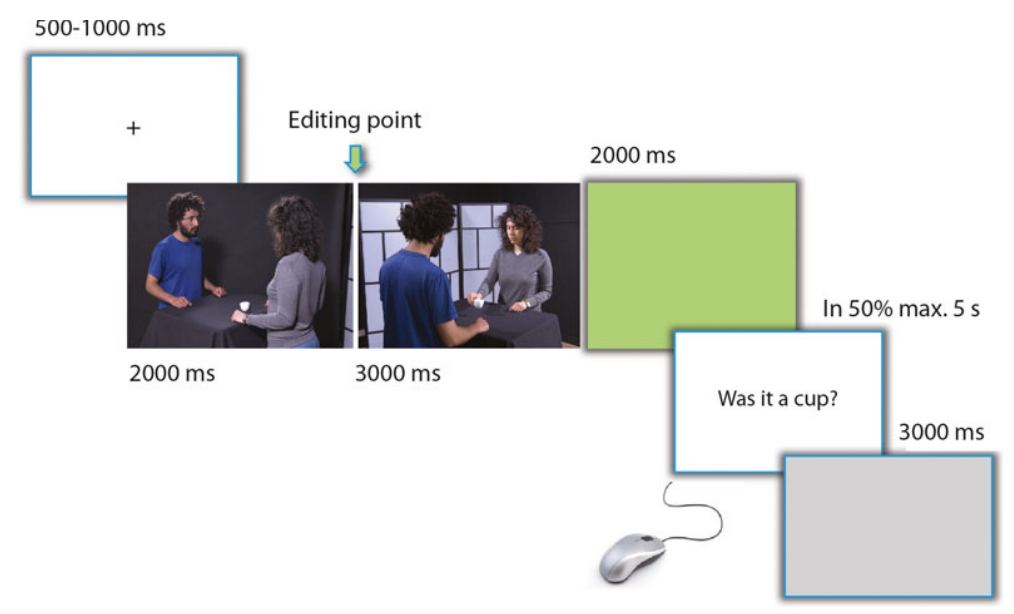

Fig. 3. Experimental paradigm during EEG recording.

displayed (guaranteeing the return of brain activity to baseline). In the other $50 \%$ of the trials, after stimulus presentation and before the gray screen, participants were presented a question on screen referring to details of the scenes in the video displayed before. An example of such questions would be: "Was the object handed over an espresso cup?" The answer had to be given by reaching out for a mouse, positioned at a distance of $15 \mathrm{~cm}$ from participants' right hand, and clicking the left button with the right index or the right button with their medium finger, allowing them to answer with "Yes" or "No." If participants gave a wrong answer or did not answer within $5 \mathrm{~s}$, they were informed that the trial was incorrect or the answer was given too slowly, and the trial was repeated. These trials served as attention control as well as action execution trial, enabling to measure brain responses during a reaching and grasping hand action (see below) (Fig. 3).

\subsubsection{Experimental design and recording details of the rating task}

The second part of the experiment consisted of a rating task programmed and executed with Eprime 2.0. Participants, still sitting in front of the screen were again shown each of the 16 video clips and for each of them were asked five different questions in five separate blocks always conducted in the same order:

1. How involved did you feel in the scene?

2. How much did you feel like the actor (the one handing over the object)?

3. How easy to watch did you find this video?

4. How natural did you find the representation of the scene in this video?

5. Which of the two last videos was longer (regarding its duration)?

Answers for Questions 1-4 were given by moving the slider on a continuous Likert Scale from 0 to 100 (representing the extremes "not at all" and "very much so"); for question 5, they clicked "Video 1" or "Video 2." The first two questions were designed to probe participants' potential feeling of involvement with the observed scene in terms 
of empathy with the actor. The third question was designed to explore how at ease participants were with the different ways of editing. The fourth question was designed to measure participants' estimation of the ecological plausibility of the different types of editing used. The last question was designed to investigate whether the different montage techniques had an influence on participants' duration estimation.

\subsubsection{Analysis}

All further processing was done using the Matlab toolbox FieldTrip (Oostenveld, Fries, Maris, \& Schoffelen, 2011).

2.3.3.1. ERP analysis: For the ERP analysis, data were filtered offline with bandpass filter $0.1-45 \mathrm{~Hz}$ and segmented into specific time epochs, each of the length of $1 \mathrm{~s}$. Data were segmented as described in the main text. Artifacts for all conditions were removed through visually inspected Independent Component Analysis as implemented in FieldTrip, considering time, topographic, and spectral distribution of the component, as well as consequent visual inspection and exclusion of all trials with still remaining artifacts. A minimum number of 50 trials for each condition were kept (fulfilled by all but two participants, who were consequently excluded from further analysis, resulting in 18 data sets being analyzed).

ERPs were computed by averaging over trials and participants using time-locked analysis over the segmented epochs as implemented in Fieldtrip. Regions of interest (ROIs) were selected on the basis of the findings of prior studies investigating ERPs due to content/structure violations (Koelsch, 2011; Kutas \& Federmeier, 2000; Kutas \& Hillyard, 1980; Lau et al., 2008; Maffongelli et al., 2015; Steinbeis \& Koelsch, 2008). In these studies, usually four ROIs were defined: anterior left, anterior right, posterior left, and posterior right. We adopted these ROIs and defined two further central Regions of Interest to allow a comparison with results of the ERD analysis when considering differences between the two editing conditions. The resulting six ROIs are indicated in Fig. A1.

To look for ERP components elicited by the two different related cuts (continuity edits and cuts-across-the-line), we selected the first second after the editing point in the videos. Furthermore, we analyzed the first second after video onset. We decided for this "baseline" as it allowed us to distinguish ERPs caused by the onset of a completely new visual stimulus (similar to an unrelated cut), from those specifically caused by a switch in camera position (related cut), before comparing continuity edits and cuts-across-the-line. ERPs were computed by averaging over trials and participants. Time windows of interest were detected based on prior literature and ERP maximum value on scalp surface was analyzed (narrow windows to avoid confounds due to overlapping components; see Fig. A2 for illustration):

Time window $1=140-190 \mathrm{~ms}$ after stimulus onset

Time window $2=180-220 \mathrm{~ms}$ after stimulus onset

Time window $3=250-380 \mathrm{~ms}$ after stimulus onset

Time window $4=400-650 \mathrm{~ms}$ after stimulus onset 
Data were extracted while averaging potential over defined ROIs and time windows. Outliers were replaced with the corresponding average values of all participants (Criteria $= \pm 2.5$ standard deviations from mean, resulting in the replacement of less than $1 \%$ of all data).

Statistics were done using ANOVAs and Duncan post hoc tests to further explore significant factors and interactions. Reported results are automatically adjusted for multiple comparisons.

2.3.3.2. ERD analysis: For the ERD analysis, the data were filtered offline with a bandpass filter of $1-30 \mathrm{~Hz}$ and segmented into specific time epochs. From observation trials (videos edited in accordance with or violating the $180^{\circ}$ rule), the $3 \mathrm{~s}$ after the editing point of the videos plus the $2 \mathrm{~s}$ of green screen were chosen. As baseline (time without any ERD expectation), 1,000 ms of gray screen ending $250 \mathrm{~ms}$ before the start of each new trial (appearance of the fixation cross) was selected in the observation trials. From all trials in which participants were asked a question, we furthermore selected segments of $1,000 \mathrm{~ms}$ starting $500 \mathrm{~ms}$ before the motor response (the button press) and ending $500 \mathrm{~ms}$ after it, in order to test for the expected ERD of the mu-rhythm during participants' own goal-directed action.

Artifact rejection was done the same way as in the ERP analysis (leading to a sufficient number of left trials for all but three participants, resulting in 17 datasets being analyzed). Frequency bands of interest were the different components of the rolandic mu-rhythm, consisting of an alpha and a beta band (see Avanzini et al., 2012). The time-frequency analysis was performed for each participant on $1.5 \mathrm{~s}$ long segments for all conditions (baseline, continuity edit, cuts-across-the-line, action execution) using Hanning tapers in $1 \mathrm{~Hz}$ intervals with a sliding time window of $0.5 \mathrm{~s}$ in the frequency range from 7 to $30 \mathrm{~Hz}$. Frequency-power coefficients were calculated by taking the average across trials for each of the 128 channels. Electrode-clusters of interest were chosen for each of the two frequency bands of interest (8-14 and 15-24 Hz) by means of a first statistical analysis comparing baseline and action execution condition in a cluster-based permutation test as implemented in FieldTrip (using dependent samples t-test statistics, Monte Carlo method, based on 500 randomizations). On the basis of the electrodes showing significant differences (see Fig. A3), for both the alpha and the beta band, two symmetrical central clusters (one in the left, the other in the right hemisphere) were chosen. As previous research has described, the source of the beta component of the mu-rhythm sometimes lying more frontal for beta bands (Stancak \& Pfurtscheller, 1996), we additionally selected one frontal cluster also showing a strong difference between conditions; see Fig. A3.

Using these clusters, specific alpha and beta frequency bands were selected for each participant following the procedure described in previous studies (Babiloni et al., 2009; Oberman, McCleery, Ramachandran, \& Pineda, 2007). The individual peak $(F)$ of attenuated frequency was determined by calculating the ratio between the frequency power in action execution trials and during baseline in $1 \mathrm{~Hz}$-wide subfrequency bands from 8$14 \mathrm{~Hz}$ for alpha and 15-24 Hz for beta. Each value was then transformed into a log-ratio and the frequency that corresponded to the log-ratio with the most negative value was 
taken as $F$. A $3 \mathrm{~Hz}$ range frequency band was chosen for each participant $(F-1 ; F+1)$ and frequency type $(\alpha$ and $\beta$ ). For the following statistical analyses, the frequency power in this $3 \mathrm{~Hz}$ range was extracted in all conditions (number of participants selected per range: $\alpha$ : 7-9 Hz: 5; 8-10 Hz: 4; 9-11 Hz: 1; 10-12 Hz: 3; 11-13 Hz: 2; 12-14 Hz: 2; ß: 16-18 Hz: 1; 17-19 Hz: 4; 18-20 Hz: 7; 20-22 Hz: 4; 21-23 Hz: 1). ROIs and specific frequency bands of each participant were then used to extract the final data from all four conditions and chosen time windows (see below). Before statistical analysis, all data were log transformed.

In order to control for effects in central alpha due to posterior alpha (commonly associated with visual attention), we furthermore extracted for each participant the respective power values from eight electrodes per hemisphere (symmetrically located, number respective central clusters) in occipital areas (electrodes 65, 66, 68, 69, 70, 7173,74 in left occipital lobe \& electrodes 76, 82, 83, 84, 88, 89, 90, 94 in right occipital lobe) using the same frequency bands as previously described.

Statistics of log-transformed data were done using ANOVAs and Duncan post hoc tests to further explore significant factors and interactions. Reported results are adjusted for multiple comparisons.

2.3.3.3. Rating task analysis: Results of the rating task showed a violation of normality not correctable through transformation. As a consequence, nonparametric statistics (Wilcoxon Signed Ranks) were used for statistical analysis, resulting in five related samples test (one for each of the questions).

Error bars in all the graphs represent standard errors.

\section{Results}

\subsection{ERP}

We analyzed ERP signatures hypothesizing to find markers of syntactic violations, modulated across conditions. Specifically, we were interested in known markers of semantic violations (frontally distributed negative deflection around $400 \mathrm{~ms}$ after stimulus onset $\rightarrow$ third or fourth time window in frontal ROIs) and syntactic violations (earlier and specifically left anterior negativity (ELAN $\rightarrow$ first or second time window in left frontal ROI) followed by a late positivity in the same region (fourth time window in left frontal ROI, see Maffongelli et al. (2015). To achieve better readability, we will stress differences/interactions involving the factor of Condition and relating to these interests.

\subsubsection{Comparison Baseline (unrelated cut)-Related Cuts}

For the first time window (140-190 ms), a $2 \times 2 \times 3$ ANOVA ( 2 levels of Condition $\times 2$ levels of Hemisphere $\times 3$ levels of Region) showed significant interactions of Condition $\times$ Hemisphere $(F(1,17)=5.47, p<.05)$ and Condition $\times \operatorname{Region}(F(2,34)=$ 4.41, $p<.05$. Post hoc tests showed that the effects were driven by left hemispheric 


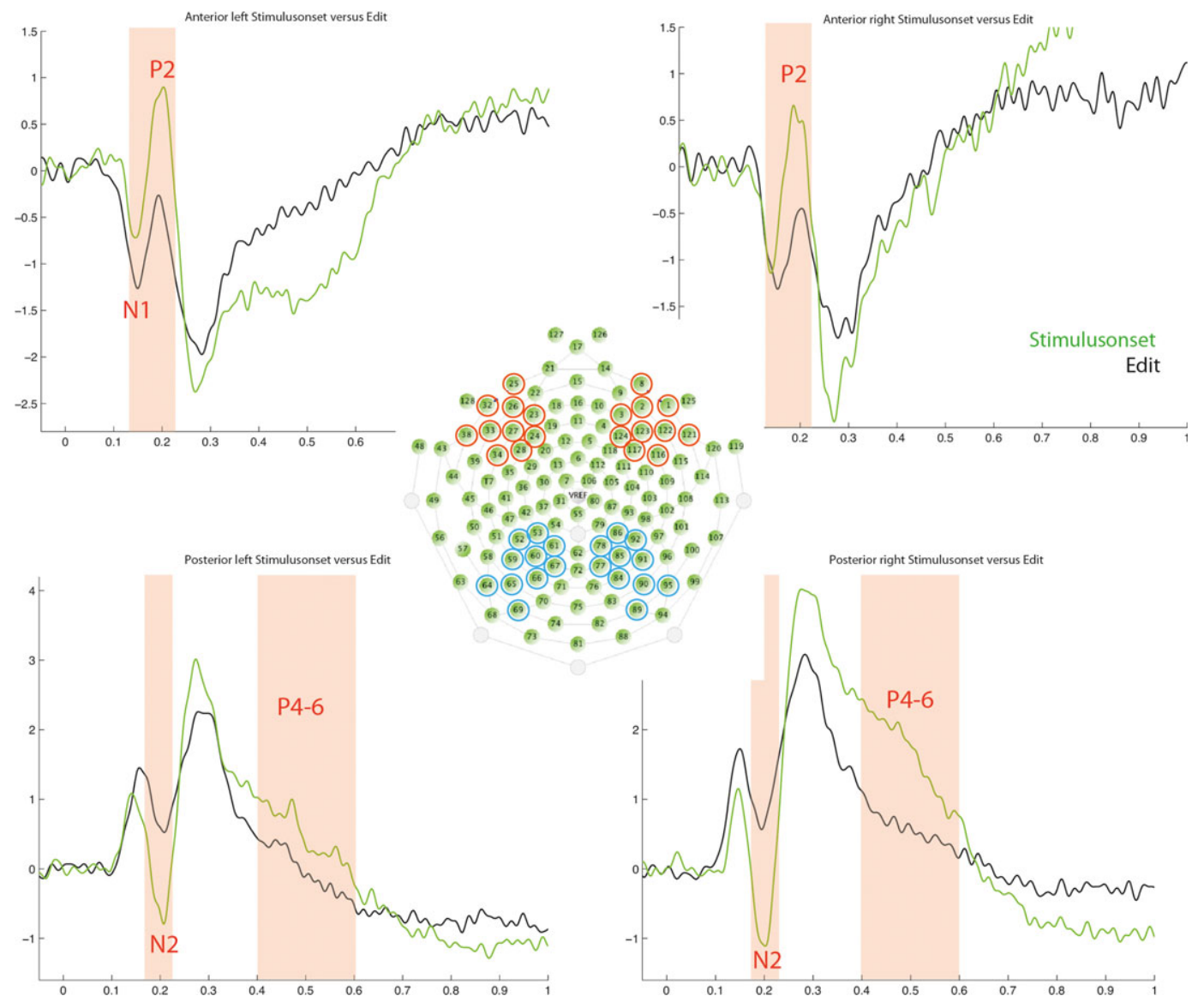

Fig. 4. Differences between baseline (unrelated cut) and related cuts in regions with significant results.

$(p<.05)$ and anterior regions $(p<.01)$, where in the grand average ERP, a negative component can be seen that is more strongly pronounced for related cuts; see also Fig. 4 (baseline left hemisphere MS: 0.037, STE: 0.047, related cuts left hemisphere MS: -0.164 , STE: 0.067; baseline anterior regions MS: -0.006 , STE: 0.081, related cuts anterior regions MS -0.212, STE: 0.092). Such differences may be interpreted as modulation of an ELAN, the expected marker for the registration of a syntactic violation (see Discussion).

For the second time window (180-220 ms), a $2 \times 2 \times 3$ ANOva ( 2 levels of Condition $\times 2$ levels of Hemisphere $\times 3$ levels of Region) showed significant main effects of Condition $(F(1,17)=10.47, p<.01$; baseline MS: -0.006 , STE: 0.042 ; related cuts MS:-0.123, STE: 0.042), Region $(F(2,34)=13.01, p<.001$; anterior regions MS: -0.666 , STE: 0.241 , central regions MS: -0.504 , STE 0.126, posterior regions: MS: 0.977 , 
STE: 0.238$)$ and a significant interactions for Condition $\times$ Region $(F(2,34)=9.72$, $p<.001)$.

Post hoc tests regarding the Condition $\times$ Region interaction showed that in anterior regions, average potential of baseline was significantly higher than that of related cuts, while in posterior regions, this relation was reversed (both $p<.01$ ); (baseline anterior regions MS: -0.245 , STE: 0.296; related cuts anterior regions MS: -1.087, STE: 0.241, baseline posterior regions MS: 0.61, STE: 0.325 , related cuts posterior regions MS 1.34, STE: 0.198).

This shows: (a) a significant difference between baseline and related cuts in anterior regions, where in the Grand Average ERP, an early positivity can be seen (in the following referred to as P2) that was more pronounced for baseline; (b) a significant difference between baseline and related cuts in posterior regions, where in the Grand Average ERP, an early negativity can be seen (in the following referred to as N2) also more pronounced for baseline, see Fig. 4. Such components are likely related to stimulus ambiguity and detection of rapid change (see Discussion).

For the third time window (250-380 ms), a $2 \times 2 \times 3$ Anova ( 2 levels of Condition $\times 2$ levels of Hemisphere $\times 3$ levels of Region) showed significant main effects for Region $(F(2,34)=45.09, p<.001$; anterior regions MS: -1.205 , STE: 0.202 , central regions MS: -0.72 , STE: 0.132 , posterior regions MS: 1.675 , STE: 0.227) only. The components causing these differences are likely to be related to face detection and working memory access, not significantly differing across our conditions (see Discussion).

For the fourth time window (400-650 ms), a $2 \times 2 \times 3$ ANOva ( 2 levels of Condition $\times 2$ levels of Hemisphere $\times 3$ levels of Region) showed significant main effects for Hemisphere $(F(1,17)=23.5, p<.001$; left hemisphere MS: -0.212 , STE: 0.092, right hemisphere: MS: 0.54, STE: 0.086), Region $(F(2,34)=12.29, p<.001$; anterior regions MS: -0.678 , STE: 0.165, central regions: MS: 0.233, STE: 0.191; posterior regions MS: 0.938, STE: 0.221$)$, and significant interactions for Condition $\times$ Hemisphere $(F(1$, $17)=4.57, p<.05)$ and Condition $\times$ Region $(F(2,34)=4.19, p<.05)$.

Post hoc tests regarding the Condition $\times$ Hemisphere showed that for both conditions, average potential was lower in the left hemisphere (for baseline $p<.001$; for related cuts, $p<.05$; baseline left hemisphere MS: -0.343 , STE: 0.132 , related cuts left hemisphere MS: -0.081 , STE: 0.113 , baseline right hemisphere MS: 0.714, STE: 0.14, related cuts right hemisphere: MS: 0.367, STE: 0.077). Post hoc tests regarding the Condition $\times$ Region interaction showed that in posterior regions, average potential in baseline was higher than average potential in related cuts $(p<.05)$ (baseline posterior regions MS: 1.299, STE: 0.319, related cuts posterior regions MS: 0.578, STE: 0.184). This indicates a significant difference between baseline and related cuts conditions in posterior regions, showing a late positivity (in the following referred to as P4-6) more pronounced for baseline; see Fig. 4. This component might be interpreted as related to the detection of a content violation or an orienting response, see Discussion below. 


\subsubsection{Comparison continuity editing-cuts-across-the-line}

For the first time window (140-190 ms), a $2 \times 2 \times 3$ ANOva ( 2 levels of Condition $\times 2$ levels of Hemisphere $\times 3$ levels of Region) showed a significant main effect only for Region $(F(2,34)=3.64, p<.05$; anterior regions MS: -0.227 , STE: 0.106 , posterior regions MS: 0.174, STE: 0.076). Such results are due to occurrence of the ERPs mentioned in a) showing no differences across these condition.

For the second time window (180-220 ms), equally, a $2 \times 2 \times 3$ ANOva ( 2 levels of Condition $\times 2$ levels of Hemisphere $\times 3$ levels of Region) showed a significant main effect only for Region $(F(2,34)=36.04, p<.001$; anterior regions MS: -1.086 , STE: 0.209, central regions: MS: -0.625 , STE: 0.12 , posterior regions MS: -1.344 , STE: 0.199). Such results are due to occurrence of the ERPs mentioned in a) showing no differences in this condition.

For the third time window (250-380 ms), a $2 \times 2 \times 3$ Anova ( 2 levels of Condition $\times 2$ levels of Hemisphere $\times 3$ levels of Region) showed significant main effects for Region $(F(2,34)=54.79, p<.001$; anterior regions MS: -1.324 , STE: 0.216 , central regions: MS: -0.784 , STE: 0.142 , posterior regions: MS: 1.719 , STE: 0.178$)$ and significant interactions for Hemisphere $\times$ Region $(F(2,34)=5.12, p<.05$; posterior left regions MS: 1.451, STE: 0.159, posterior right regions: MS: 1.985, STE: 0.277) only. Such results are due to occurrence of the ERPs mentioned in a) showing no differences in this condition.

For the fourth time window (400-650 ms), a $2 \times 2 \times 3$ ANOva ( 2 levels of Condition $\times 2$ levels of Hemisphere $\times 3$ levels of Region) showed significant main effects for Hemisphere $(F(1,17)=6.25, p<.05$; left hemisphere MS: -0.097 , STE: 0.12, right hemisphere: MS: 0.408, STE: 0.099), Region $(F(2,34)=10.12, p<.001$; anterior regions MS: -0.427 , STE: 0.139 , central regions MS: 0.554 , STE: 0.169 , posterior regions MS: 0.38, STE: 0.188) and a significant interaction of Condition $\times$ Hemisphere $\times$ Region $(F(2,34)=3.91, p<.05)$. Post hoc tests regarding the Condition $\times$ Hemisphere $\times$ Region interaction showed that the continuity edits significantly differed from cuts-across-the-line in left anterior $(p<.01)$ as well as right central $(p<.05)$ regions (continuity edit anterior left MS: -0.571 , STE: 0.171 , cutsacross-the-line anterior left MS: -1.104 , STE: 0.291, continuity edit central right MS: 0.514, STE: 0.252, cuts-across-the-line central right MS: 0.892, STE: 0.25). This shows: (a) a significant difference between the two conditions in a late left anterior positivity (P4-6 ant left) stronger for the continuity edit condition; and (b) a late right central positivity (P4-6 cen right), stronger for the cuts-across-the-line condition (see Fig. 5). These components might be related to (a) a remapping processes following the detection of a syntactic violation; and (b) perceptual processes reaching awareness, see Discussion.

\subsubsection{EEG-ERD analysis}

We measured and analyzed ERD of central mu- and occipital alpha rhythms hypothesizing to find modulations across conditions indicating either differences in sensorimotor processing (central mu-rhythm) or in attention (occipital alpha rhythm). 


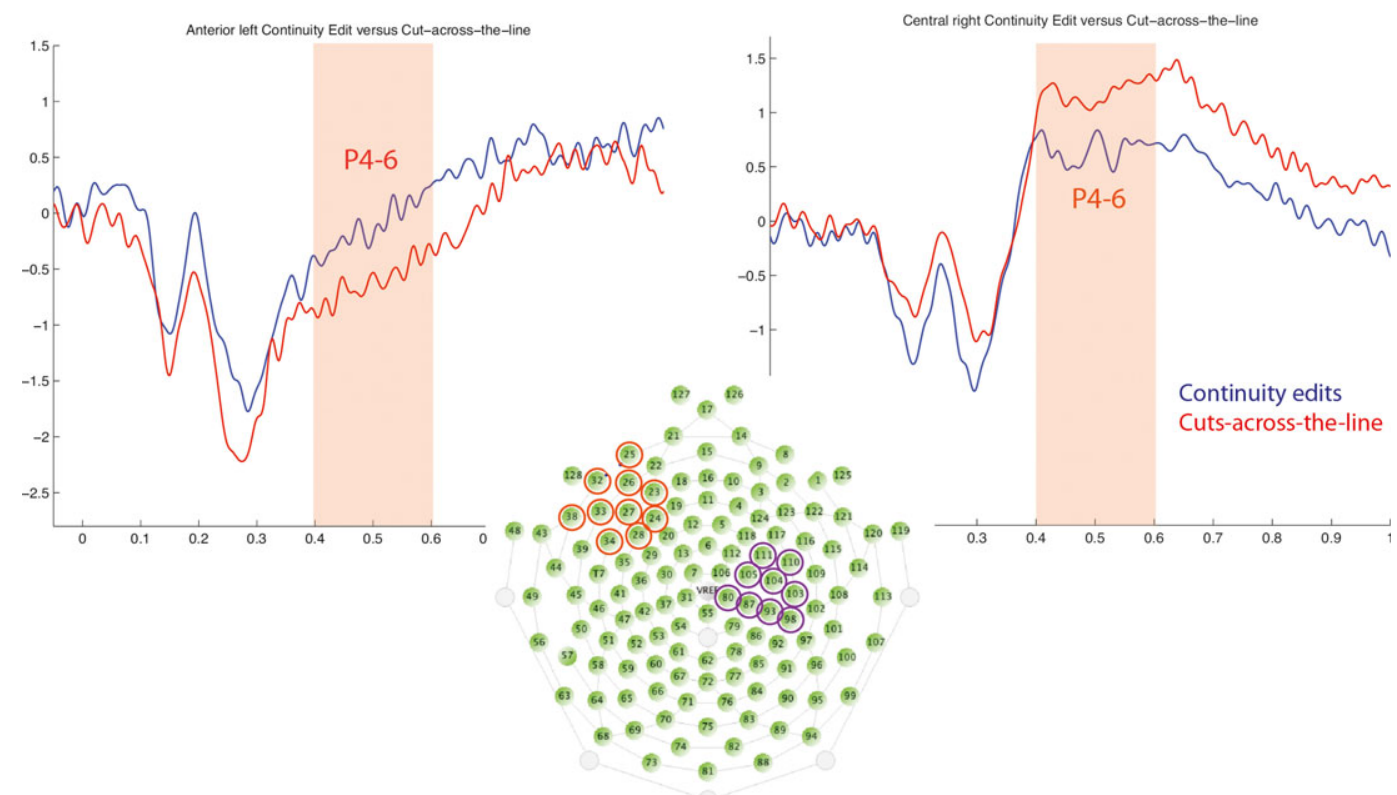

Fig. 5. Differences between continuity edits and cuts-across-the-line in regions with significant results.

We first executed a $4 \times 2$ (alpha-power) and a $4 \times 3$ (beta-power) ANOva to test for the typical desynchronization pattern expected for the mu-rhythm in general, that is a significant difference between participants' own goal-related action execution (segments extracted from participants button-press when answering questions), action observation (here: movie-clips), and baseline (baseline $>$ action observation $\geq$ action execution). Results showed differences in all band ranges and regions, justifying our further analysis (see Fig. 6). Furthermore, beta bands in central regions showed stronger desynchronization than in frontal regions: $F(2,32)=16.66, p<.001$. For the following analysis, we therefore analyzed the ERD time course separately for frontal and central regions. For reasons of clarity, complete results of these analyses can be found in the Appendix.

In the further analyses, we explored differences in the time course between the two different cut conditions. The main effect of Time in all three analyses reflected only the expected difference between desynchronization (during video) and resynchronization (after video) (see Fig. 7). For clarity of the manuscript, the precise results of this effect can be found in the Appendix.

For central alpha power values, the $2 \times 2 \times 5$ ANOva ( 2 levels of Condition [continuity edit and cuts-across-the-line], 2 levels of Hemisphere [left vs. right], and 5 levels of Time $[5 \mathrm{~s}$ from cut on] ) showed a significant main effect of Time $(F(4,64)=26.10$, $p<.001)$ as well as a significant interaction Condition $\times \operatorname{Hemisphere}(F(1,16)=6.22$, $p<.05)$. 
Central Alpha and Frontal Beta Power Differences

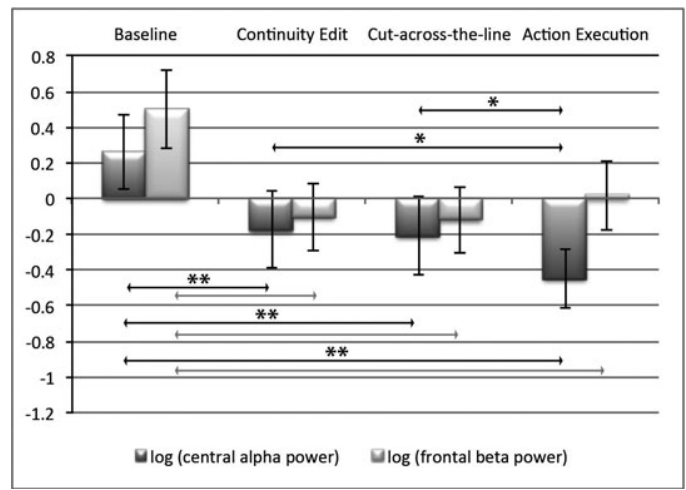

Central Beta Left and Right Hem. Power Differences

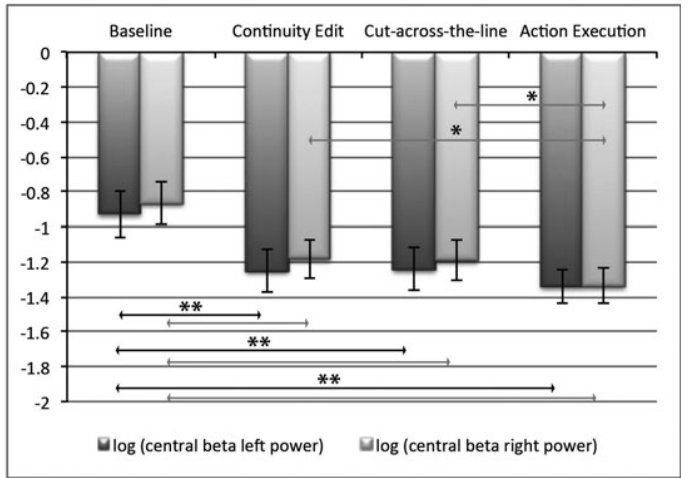

Fig. 6. Results of first analysis assessing ERD of the different components of the mu-rhythm during movie watching (action observation after edit) and during the participants' executed hand action. Condition effect for alpha range: $F(3,48)=20.67, p<.001$, for beta ranges: $F(3,48)=25.63, p<.001$. Results show typical desynchronization (indicated by the significant difference from baseline) for action observation and action execution for all band ranges and regions $(p<.001)$, with significant differences between observation conditions and action execution for central alpha and central right beta power $(p<.01 / p<.05)$. Furthermore, beta bands in central regions showed stronger desynchronization than in frontal regions: $F(2,32)=16.66$, $p<.001 . *=<0.05 ; * *=<.001$.

Post hoc comparisons further investigating the significant Condition $\times$ Hemisphere interaction showed that the significant differences occurred between left and right hemisphere for continuity edit conditions (with the left hemisphere being more strongly desynchronized than the right one, $p<.001)$ and between continuity edits and cuts-across-theline in the right hemisphere (with continuity edits being less desynchronized than cutsacross-the-line $p<.01$ ) (continuity edits left hemisphere MS: -0.3 , STE: 0.082, continuity edits right hemisphere MS: -0.273 , STE: 0.079 , cuts-across-the-line left hemisphere MS: -0.289 , STE: 0.08, cuts-across-the-line right hemisphere MS: 0.291, STE: 0.078); see Fig. 8. These results indicated a different processing of continuity edits and cutsacross-the-line by the sensorimotor system, with cuts-across-the-line correlating with a effector and hemisphere unspecific desynchronization. For further details, see Discussion below.

For central beta power values, the $2 \times 2 \times 5$ ANOva (Condition $\times$ Hemisphere $\times$ Time $)$ showed a significant main effect of Time only $(F(4,64)=23.53$, $p<.001)$; see Fig. 7 .

For frontal beta power values, the $2 \times 5$ ANOVA ( 2 levels of Condition (continuity edits and cuts-across-the-line) and 5 levels of Time [ $5 \mathrm{~s}$ from cut on]) showed a significant main effect of Time only $(F(4,64)=17.36, p<.001$; see Fig. 7).

To control for similar effects in occipital regions (an alpha rhythm associated with attention), we repeated the analysis just described for central and frontal alpha in occipital electrodes. Results showed significant effects only as to be expected from visual stimulation in general (significant difference to baseline in the first analysis, de- and 


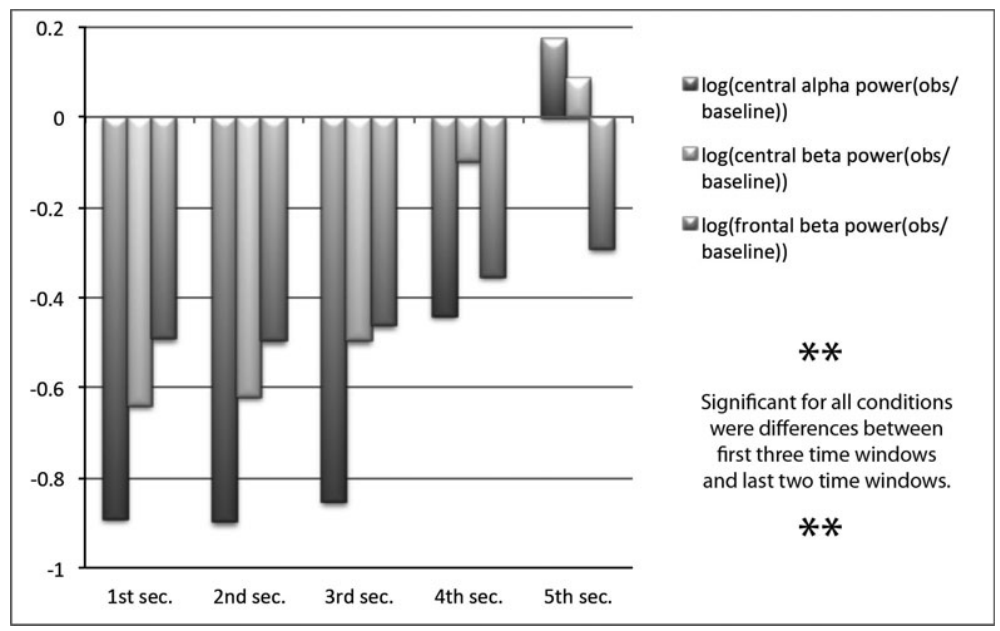

Fig. 7. Time course differences in central alpha, central beta, and frontal beta power over $5 \mathrm{~s}$ after an edit ( $3 \mathrm{~s}$ of movie showing goal related action, $2 \mathrm{~s}$ resynchronization phase). Time effect in central alpha: $F(4,64)=26.1, p<.001$, central beta: $F(4,649=23.53, p<.001$, frontal beta: $F(4,64)=17.36, p<.001$. Differences between desynchronization and resynchronization periods were significant in all bands and regions $(p<.01$ for central alpha, $p<.001$ for central and frontal beta).

resynchronization in the second analysis, see Fig. 9). This means that our results do not support a modulation of attention by editing techniques. For details of the results, please see Appendix; for a more detailed interpretation, see Discussion.

\subsubsection{Rating task}

Only the results of question 4 of the rating task ("How natural did you find the representation of the scene in this video?") did reveal significant differences between observation conditions. For this question, participants rated videos with a continuity edit as more natural in their representation of the scene than videos including a cuts-across-the-line $(N=17, T=25, Z=2.22, p<.05)$ (continuity edit MS: 34.912, STE: 5.313, cut-acrossthe-line MS: 30.257, STE: 5.221); see Fig. 10.

Results of the other questions can be found in the Appendix.

\section{Discussion}

Previous research led to the hypothesis that continuity edits and cuts-across-the-lines differ in terms of updating processes taking place in visual systems possibly due to a modulation of visual attention related to the salience of a violation of perceptual habits (Magliano \& Zacks, 2011). According to this hypothesis, the presence of these updating processes would enable the smooth perception of the moving images in continuity edits, while their attention-driven suppression in cuts-across-the-line would cause their greater 
Central Alpha Power

Condition ${ }^{*}$ Hemisphere Interaction

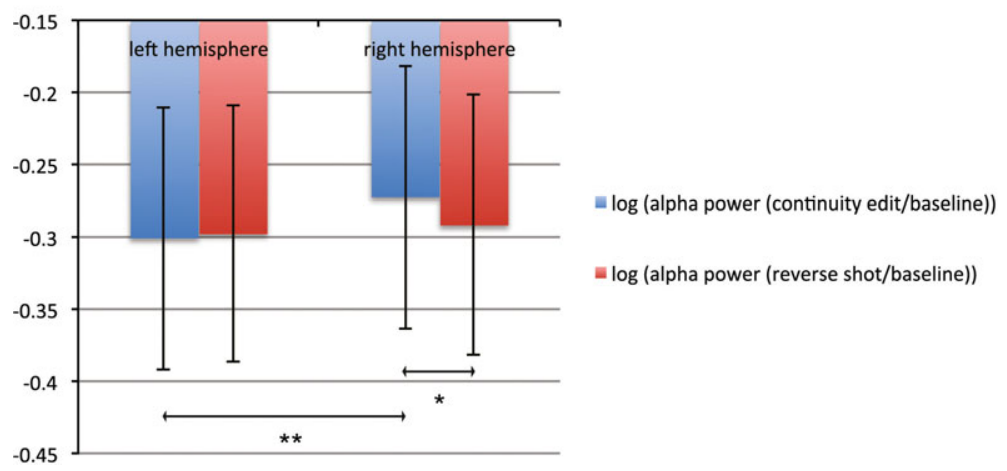

Fig. 8. Central alpha power results of the Condition $\times$ Hemisphere interaction: $F(1,16)=6.22, p<.05$. Indicated are significant differences between alpha power for continuity edits in left and continuity edits in right hemisphere $(p<.001)$, as well as between continuity edits and cuts-across-the-line in right hemisphere $(p<.01$. $)$

Occipital Alpha Power Differences

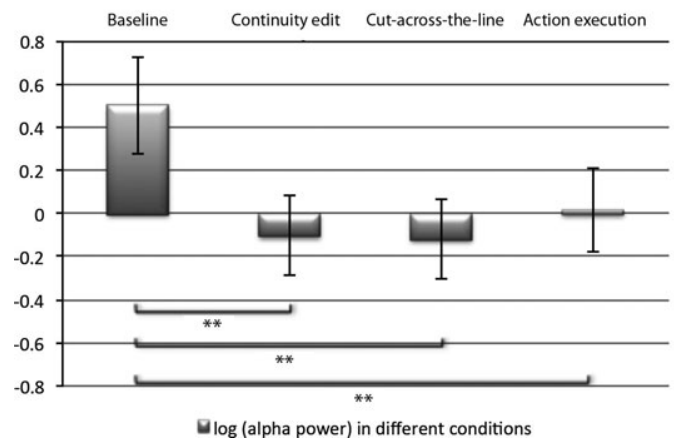

Timecourse Occipital Alpha Power Observation Conditions

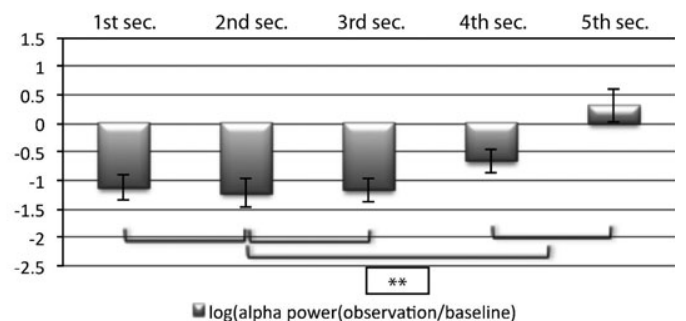

Fig. 9. Differences in occipital alpha. The $4 \times 2$ ANova shows a main effect of Condition only $F(3,48)=21.44, p<.001$. Post hoc comparisons show that alpha frequency power for baseline was significantly higher than for all other conditions $(p<.001)$. The $2 \times 2 \times 5$ ANOvA showed a significant effect of Time only. $F(4,64)=23.93, p<.001)$. Post hoc tests show that significant differences occurred between all time windows during video observation (desynchronization phase) and all time windows after video observation (resynchronization phase) (all $p<.05)$. ** $\leq 0.01$.

perceptual saliency. Our findings do support and refine this hypothesis with respect to differences among editing conditions in later stages of stimulus processing related to the detection of a violation of perceptual anticipations. However, our results do not support this hypothesis with regard to a role of an attention-driven top-down modulation. Instead, they indicate a possible role for action-observation networks that should be further examined. The following paragraphs will go through all the significant effects observed, while in the summary, the most relevant findings for the hypothesis will be highlighted. 
How natural did you find the representation of the scene out of the video

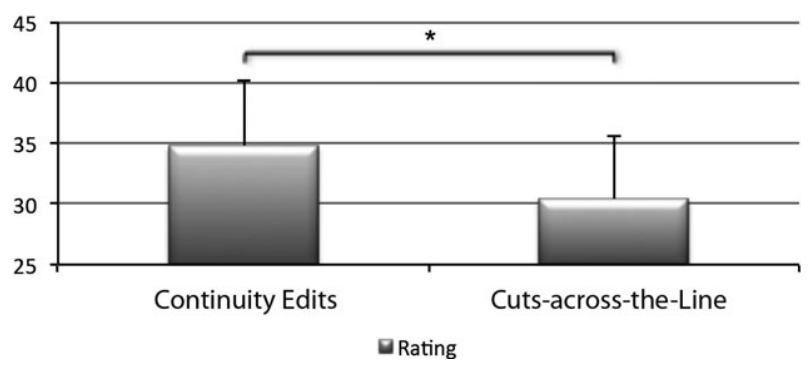

Fig. 10. Results of the rating task question: "How natural did you find the representation of the scene in this video?," showing that videos containing a continuity edit were judged as more naturally representing the scene than videos containing a cut-across-the-line. Results of Wilcoxon Signed Rank: $N=17, T=25$, $Z=2.22, p<.05 . *=<0.05$;

\subsection{ERP results}

An ERP analysis was performed to look for markers of semantic or syntactic violation detection and processing, as known from other tasks such as language, music, and action sequence processing.

For the first time window (140-190 ms), we found an amplitude increase of an early anterior negativity (N1) for all conditions, which was especially pronounced in the left hemisphere. Such timing and location fits to the component previously suggested to indicate the detection of a syntactic violation (in language, music, and observation of action sequences; see Maffongelli et al., 2015). The amplitude of such component was higher for related cuts (in comparison to the baseline $=$ unrelated cut).

Our results of the first time window thus suggest that while both the visual onset of a video as well as the visually different stimulus after a cut elicit an ERP associated with the detection of a syntactic violation, such violations are perceived as stronger after a related cut. This indicates that it is not solely the difference between consecutive images (which would be stronger for the baseline as a switch from a black screen to the shot) that is driving this response, but rather an unexpected event (here a perspective change) in the ongoing action. It must be noted though, that this finding might be due to perspective changes in the observed scenes, while not being necessarily specific for actions. Future research is needed to investigate this possibility. No significant differences between the two cut conditions (continuity edits vs. cuts-across-the-line) were found in this time window.

In the second time window (180-220 ms), we found significantly higher potential for baseline (unrelated cut) than for related cuts in anterior regions as well as significantly lower potential for unrelated cuts than for related cuts in posterior regions. Together with the descriptive information of the Grand Average ERP, this shows an amplitude increase of an early anterior positivity (P2) as well as early posterior negativity (N2) for unrelated cuts. 
Previous research has correlated the amplitude increase of P2 with decreasing stimulus ambiguity (Kornmeier \& Bach, 2012). The visual change produced by a stimulus onset is a clear event of a rather non-ambiguous character. The change caused by a cut involving the editing of two shots filmed from different camera positions, in contrast, is a less easily interpretable event. More precisely, the same scene is still observed although the camera has been displaced, thus breaking visual continuity. It is possible that these kinds of stimuli elicited a response signaling ambiguity, as some visual features did not change, while others changed.

Interpretations of the differences found in posterior regions (N2) could reflect the detection of a mismatch due to sudden changes in bottom-up visual information, stronger for the change from black screen to scene onset (baseline/unrelated cut), see for example Kimura, Schröger, \& Czigler (2011).

Neither in the second time window difference between continuity edits and cutsacross-the-line condition was found.

For the third time window, no effects involving the main factor of condition were found. Found effects for Region are due to the occurrence of fronto-central negativities around $300 \mathrm{~ms}$ after stimulus onset and temporal/parietal positivities around $300 \mathrm{~ms}$ after stimulus onset as visible in Fig. A2. Fronto-central negativities with this occurrence in time have been reported to be associated with the detection of faces (Barrett, Rugg, \& Perrett, 1988; Debruille, Brodeur, \& Franco Porras, 2012; Debruille, Pineda, \& Renault, 1996). Faces are indeed present in all of our stimuli conditions. Positivities (in temporal or parietal regions) around $300 \mathrm{~ms}$ after stimulus onset have been associated with working memory activities indicating a perceived change of the environment (Donchin \& Coles, 1988; Patel \& Azzam, 2005; Polich, 2007). The fact that we did not find significant differences between conditions here is likely to indicate that face detection and working memory access do not significantly differ among our conditions.

For the fourth time window (400-650 ms), we found a significantly higher potential for baseline (unrelated cut) than for related cuts in posterior regions. Together with the descriptive information of the Grand Average ERP, this showed an amplitude increase of a late posterior positivity (P4-6) for baseline. There are several ways of interpreting this finding. Firstly, as reported above, late posterior positivities have been described in language research to correlate with syntactic violations. However, it was also reported that strong content violations correlate with a pronounced posterior P4-6 (van Meerendonk, Kolk, Vissers, \& Chwilla, 2012), possibly indicating a contextual update. It can therefore be hypothesized that the change from black screen to video onset elicits the registration of a content-related semantic violation, leading to a contextual update indicated by a pronounced posterior P4-6. Secondly, it might be possible that the observed P4-6 has to be interpreted as a slow cortical potential, since it has been observed as being associated with an orienting response. Indeed, Francuz and Zabielska-Mendyk (2013) found that unrelated cuts elicit stronger SCP, positive in parietal regions, 448-648 ms after editing point. It has to be noted though that our study did not show the related negative potential in frontal regions reported by Francuz and Zabielska-Mendyk (2013). Future research has to further explore these possibilities. 
More importantly, comparing continuity edits and cuts-across-the-line conditions within this time window, we found two significant differences: Firstly, we measured significantly higher potential (P4-6) for continuity edits than for cuts-across-the-line in left anterior regions. As reported above, recent studies exploring content and structure violations in action observation observed a late P4-6 in left anterior regions following syntactic violations (indicated by early left anterior negativities), associated with postperceptual processes possibly serving an adjustment to the detected violation, likely helping to overcome the change without reaching visual awareness (Maffongelli et al., 2015). Indeed, in our data, all conditions show an early anterior negativity, though this component is not different in amplitude when comparing continuity edits and cuts-across-theline. Interestingly, the late P4-6 component indicating updating or reanalyzing is stronger for the continuity editing condition. Remember that Magliano and Zacks (2011), suggested that such updating processes might be suppressed for edits not following continuity rules. While our results cannot decide if we are dealing with a suppression or an enhancement here, they do support the differences between conditions regarding such updating processes.

Furthermore, in central right regions, we found a significantly higher potential (P4-6) for cuts-across-the-lines when compared to continuity edits. Previous ERP studies investigating the neural correlates of change blindness found a pronounced late positivity (300$700 \mathrm{~ms}$ ) over central parietal lobes only when a change was detected (Koivisto \& Revonsuo, 2003; Niedeggen, Wichmann, \& Stoerig, 2001). Koivisto and Revonsuo (2003) suggested that these changes are likely to be associated with postperceptual processes such as conscious evaluation of change and decision-making. According to the authors, this ERP does not specifically correlate to the subjective experience of seeing but more broadly to perceiver's other beliefs about the experience with the seen event. (Koivisto \& Revonsuo, 2003, p. 428). The late right central P4-6 more strongly evoked by cutsacross-the-line could thus be associated with the spectators' reflection about the conditions of their own perception, including standard implicit anticipation of forecoming events.

Interestingly also, a late positivity in central parietal regions has also been described in studies investigating action monitoring and error detection for one's own actions (Falkenstein, Hoormann, Christ, \& Hohnsbein, 2000; Niewenhuis, Ridderinkhof, Blom, Band, \& Kok, 2001; Ruchsow, Spitzer, Grön, Grothe, \& Kiefer, 2005) Specifically, it has been reported that a late positive ERP amplitude in centro-parietal electrodes gets strongly pronounced upon the detection of errors in one's own actions. Our results suggest the hypothesis that this positivity might represent a more general phenomenon related to the awareness of an observed action - either one's own or another's - not matching sensorimotor predictions. Further research is needed to investigate this possibility.

\subsection{ERD results}

ERD Analysis was performed to look for modulations of central mu-rhythm and occipital alpha rhythm (control) as the two most common markers for the sensorimotor 
responses during action observation (central mu-rhythm) and visual attention (occipital alpha rhythm).

In general, the ERD results showed the expected desynchronization of the mu-rhythm during action observation and execution in both alpha and beta bands: When analyzing frequency power of both bands in selected electrodes during the second second of action observation as well as during the second in which participants performed the button press, a significant desynchronization during action observation as well as during action execution was found.

Further investigation of the whole time course of mu-rhythm desynchronization and resynchronization for observation conditions in beta bands did not show any difference between conditions (continuity edits vs. cuts-across-the-line). In contrast, in the alpha band, a significant Condition $\mathrm{x}$ Hemisphere interaction was detected. Post hoc tests revealed that for continuity edits, the significant difference occurred between hemispheres, with the left hemisphere showing stronger ERD than the right hemisphere. For cuts-across-the-line, however, ERD was equal in both hemispheres, leading to a significant difference between continuity edits and cuts-across-the-line in the right hemisphere.

When interpreting our findings, we would like to draw on the fact that a camera displacement of $180^{\circ}$ (cuts-across-the-line) causes a mirroring of the original spatial relations of the viewer to the scene: What has been presented as left before becomes right after the cut. Previous studies reported that during the observation of hand actions, mu suppression in the alpha band is larger in the hemisphere contralateral to the observed moving hand (Perry \& Bentin, 2009; for similar findings in fMRI, see also Shmuelof \& Zohary, 2005). We suggest that the left-right reversal caused by the cut-across-the-line might cause a short orientation deficit, disturbing the clear identification of the observed hand performing the action. In consequence, for cuts-across-the-lines, no clear contralateral lateralization of the ERD might occur, but rather both hemispheres should show equally strong ERD - as indeed, it was found in the present experiment.

Results of analyses of alpha power in occipital regions do not reveal comparable results, supporting the notion that alpha ERD in occipital cortices is discriminable from alpha ERD in motor areas. Furthermore, they also do not reveal general differences in occipital alpha between conditions in general, which means that our data do not support the suggestion that the different stimuli differently modulate visual attention, therefore being processed differently. On the contrary, it might be possible that the modulation of central alpha influences postperceptual processing as it is also reflected in the different late ERP responses to the two conditions.

As a note of caution though, it has to be stressed that the presented results only hint in this direction, as there are a couple of arguments that question our hypothesis of a prominent role of the motor cortex or action observation networks. Firstly, it can be argued that our way of selecting clusters and ROIs, which is exclusively based on previous research, is priming the data. Future research should thus look at different locations, possibly also employing source localization to strengthen our suggestion about the involvement of the motor cortex and action observation processes. Secondly, we did not find a significant lateralization for the actual motor execution condition. Previous studies 
have shown that such lateralization is not always present, and it could be that it is more pronounced in the observation condition, as shown in our study. Future research, possibly involving a replication of our study looking at left hand actions, is thus needed to further support our hypothesis. Lastly, it should be further investigated whether there is a link between the ERD results and the ERP results in central regions by designing correlational studies.

The main findings of our study can thus be summarized as follows. First, related cuts in general, no matter whether continuity edits or cuts-across-the-line, correlate with modulations of early ERP components. These markers were previously associated with the detection of syntactic violations in general and of actions in particular, as well the detection of a sudden visual change. This supports the idea that related cuts violate certain expectations regarding the flow of the event streams or actions depicted. Remarkably, however, no differences between continuity edits and cuts-across-the-line were found for early components, suggesting that early perceptual detection does not yet differentiate the two conditions.

Second, our results show a late anterior left positivity that is stronger for continuity edits than cuts-across-the-line. In previous research, this component was interpreted as most likely representing updating processes after the detection of syntactic violations serving the reanalysis of the scene (see Maffongelli et al., 2015). Our results thus seem to support Magliano and Zacks (2011), who suggested differences between conditions regarding postperceptual updating mechanisms while at the same time, further specifying those as processes elicited by the detection of syntactic violations.

Third, we found a late central right positivity (P4-6) more strongly pronounced for cuts-across-the-line. Previous research on change blindness found this component occurring only when a change was detected and suggested an association of this component with an access to reflexive consciousness, marking the violation of own perceptual expectations. Our research thus indicates a role of such access in spectators' different experiences of the two edits.

Interestingly, the described differences are not mirrored in significant differences in the ERD in the alpha frequency band in posterior regions, associated with visual attention, as Zacks and Magliano's suggestion regarding a crucial role of attention in the modulation of responses would have predicted. However, we did find a difference in hemispheric lateralization of the ERD of the central mu rhythm between conditions, possibly reflecting a sensorimotor violation elicited by cuts-across-the-lines.

Further research is needed to investigate these hypotheses, ideally also including stimuli that do not involve actors performing a goal-directed action, as such stimuli might or might not recruit other mechanisms, rendering our findings as more or less specific for the domain of observed actions.

\section{Conclusion}

We used high-density EEG recording during the observation of highly controlled stimuli, to explore how the brain processes different types of film editing. Empirical research 
about the perception of edits has shown that edits complying with the $180^{\circ}$ rule correlate with a smooth perception of the represented events, with spectators often not even detecting the cut when explicitly asked to do so. Cuts-across-the-line, on the other hand, which violate this rule, have been reported to be detected much more often. As an explanation of these findings, it has been suggested that the continuity edits would be processed via the use of spatial updating processes, masking their disruptive nature, that were suppressed for cuts-across-the lines due to attentional downregulation (Zacks \& Magliano, 2011).

Our results seem to further corroborate the suggestion of different processing mechanisms involved in processing movie cuts. The main differences between the two types of cuts concern later postperceptual processes that seem to be modulated by the degree of the deviation of the stimuli from real-world phenomena. More specifically, our ERP findings suggest that the differences are picked up and further processed as syntactic violations by networks possibly shared for language, music, and action processes. However, our findings do not support the hypothesis that the differences in processing routes are mainly linked to visual attention as we could not detect any modulations of occipital alpha frequencies between conditions. On the contrary, our results found specific modulations of the central mu rhythm ERD as an indicator of sensorimotor activity, suggesting that sensorimotor networks might play an important role. These findings shed new light on processes involved in film perception and should be considered when explaining and especially when further exploring spectators' different experience of different kinds of cuts.

\section{Acknowledgments}

This work was supported by the EU grant Towards an Embodied Science of InterSubjectivity (TESIS, FP7-PEOPLE-2010-ITN, 264828) to K.H., S.U., and V.G.; Marie Sklodowska-Curie Grant 657605 of the European Union's Framework Programme, Horizon 2020 to S.U.; and by a grant of Chiesi Foundation to V.G. We thank Pia de Stefano for her most valuable help in stimuli production and data registration, and Chris and Uta Frith as well as Joerg Fingerhut for their most helpful comments.

\section{References}

Avanzini, P., Fabbri-Destro, M., Dalla Volta, R., Daprati, E., Rizzolatti, G., \& Cantalupo, G. (2012). The dynamics of sensorimotor cortical oscillations during the observation of hand movements: An EEG study. PLoS ONE, 7(5), e37534. doi:10.1371/journal.pone.0037534

Babiloni, C., Del Percio, C., Rossini, P. M., Marzano, N., Iacoboni, M., Infarinato, F., Lizio, R., Piazza, M., Pirritano, M., Berlutti, G., Cibelli, G., \& Eusebi, F. (2009). Judgment of actions in experts: A highresolution EEG study in elite athletes. NeuroImage, 45, 512-521.

Barrett, S. E., Rugg, M. D., \& Perrett, D. I. (1988). Event related potentials and the semantic matching of familiar and unfamiliar faces. Neuropsychologia, 26, 105-117. 
Bordwell, D. (1985). Narration in the fiction film. Madison: University of Wisconsin Press.

Bordwell, D., \& Thompson, K. (2006). Film art: An introduction. New York: McGraw-Hill.

Calvo-Merino, B., Glaser, D. E., Grèzes, J., Passingham, R. E., \& Haggard, P. (2005). Action observation and acquired motor skills: An FMRI study with expert dancers. Cerebral Cortex, 15, 1243-1249.

Calvo-Merino, B., Grèzes, J., Glaser, D. E., Passingham, R. E., \& Haggard, P. (2006). Seeing or doing? Influence of visual and motor familarity in action observation. Current Biology, 16, 1905-1910.

Casetti, F. (2015). The Lumière Galaxy. Seven Key Words for the Cinema to Come. New York: Columbia University Press.

Cutting, J. E. (2005). Perceiving scenes in film and in the world. In J. D. Anderson \& B. F. Anderson (Eds.), Moving image theory: Ecological considerations (pp. 9-27). Carbondale, IL: Southern Illinois University Press.

Debruille, J. B., Brodeur, M. B., \& Franco Porras, C. (2012). N300 and social affordances: A study with a real person and a dummy as stimuli. PLoS ONE publication without paper version, Published: October 31, 2012 http://dx.doi.org/10.1371/journal.pone.0047922

Debruille, J. B., Pineda, J., \& Renault, B. (1996). N400-like potentials elicited by faces and knowledge inhibition. Brain Research. Cognitive Brain Research, 4, 133-144.

Donchin, E., \& Coles, M. G. H. (1988). Is the P300 component a manifestation of context updating? Behavioral and Brain Sciences, 11, 357-374.

Falkenstein, M., Hoormann, J., Christ, S., \& Hohnsbein, J. (2000). ERP components on reaction errors and their functional significance: A tutorial. Biological Psychology, 51, 87-107.

Francuz, P., \& Zabielska-Mendyk, E. (2013). Does the brain differentiate between related and unrelated cuts when processing audiovisual messages? An ERP study. Media Psychology, 16, 461-475

Gallese, V. (2007). Before and below Theory of Mind: Embodied simulation and the neural correlates of social cognition. Philosophical Transactions of the Royal Society of London B, 362, 659-669.

Gallese, V., \& Sinigaglia, C. (2011). What is so special about embodied simulation? Trends in Cognitive Sciences, 15, 512-519.

Geiger, S., \& Reeves, B. (1993). The effects of scene changes and semantic relatedness on attention to television. Communication Research, 20, 155-175.

Germeys, F., \& D'Ydewalle, G. (2007). The psychology of film: Perceiving beyond the cut. Psychological Research, 71, 458-466.

Hamilton, A., \& Grafton, S. (2009). Repetition suppression for performed hand actions revealed by fMRI. Human Brain Mapping, 30, 2898-2906.

Heimann, K., Umilta, M. A., Guerra, M., \& Gallese, V. (2014). Moving mirrors: A high density EEG study investigating the effect of camera movements on motor cortex activation during action observation. Journal of Cognitive Neuroscience, 26, 2087-2101.

Kimura, M., Schröger, E., Czigler, I., \& Ohira, H. (2010a). Human visual system automatically encodes sequential regularities of discrete events. Journal of Cognitive Neuroscience, 22, 1124-1139. doi: 10.1162/ jocn.2009.21299

Koelsch, S. (2011). Toward a neural basis of music perception - a review and updated model. Frontiers in Psychology, 2, 110.

Koivisto, M., \& Revonsuo, A. (2003). An ERP study of change detection, change blindness, and visual awareness. Psychophysiology, 40, 423-429.

Kornmeier, J., \& Bach, M. (2012). Ambiguous figures - what happens in the brain when perception changes but not the stimulus. Frontiers of Human Neuroscience, 6(51). doi: 10.3389/fnhum.2012.00051

Kutas, M., \& Federmeier, K. (2000). Electrophysiology reveals semantic memory use in language comprehension. Trends in Cognitive Sciences, 4, 463-470.

Kutas, M., \& Hillyard, S. (1980). Reading senseless sentences: Brain potentials reflect semantic incongruity. Science, 207, 203-205.

Lang, A., Geiger, S., Strickwerda, M., \& Sumner, J. (1993). The effects of related and unrelated cuts on viewers memory for television: A limited capacity theory of television viewing. Communication Research, 20, 4-29. 
Lau, E. F., Phillips, C., \& Poeppel, D. (2008). A cortical network for semantics: (de)constructing the N400. Nature Review Neuroscience, 9, 920-933.

Maffongelli, L., Bartoli, E., Sammler, D., Koelsch, S., Campus, C., Olivier, E., Fadiga, L., \& D'Ausilio, A. (2015). Distinct brain signatures of content and structure violation during action sequence observation. Neuropsychologia, 75, 30-39.

Magliano, J. P., \& Zacks, J. M. (2011): The impact of continuity editing in narrative film on event segmentation. Cognitive Science - A Multidisciplinary Journal, 35, 1489-1517.

Matran-Fernandez, A., \& Poli, R. (2015). Event-related potentials induced by cuts in feature movies and their exploitation for understanding cut efficacy, 7th International IEEE.

van Meerendonk, N., Kolk, H., Vissers, C., \& Chwilla, D. (2012). Monitoring in language perception: Mild and strong conflicts elicit different ERP patterns. Journal of Cognitive Neuroscience, 22, 67-82.

Murch, W. (1992). In the blink of an eye. A perspective on film editing. 2nd Ed. Los Angeles: Silman-James Press.

Muthukumaraswamy, S., \& Johnson, G. (2004). Changes in rolandic mu rhythm during observation of a precision grip. Psychophysiology, 41, 152-156.

Muthukumaraswamy, S. D., Johnson, B. W., \& McNair, N. A. (2004). Mu rhythm modulation during observation of an object-directed grasp. Cognitive Brain Research, 19, 195-201.

Niedeggen, M., Wichmann, P., \& Stoerig, P. (2001). Change blindness and time to consciousness. European Journal of Neuroscience, 14, 1719-1726.

Niewenhuis, S., Ridderinkhof, R., Blom, J., Band, G., \& Kok, A. (2001). Error-related brain potentials are differentially related to awareness of response errors: Evidence from an antisaccade task. Psychophysiology, 38, 752-760.

Oberman, L. M., McCleery, J. P., Ramachandran, V. S., \& Pineda, J. A. (2007). EEG evidence for mirror neuron activity during the observation of human and robot actions: Toward an analysis of the human qualities of interactive robots. Neurocomputing, 70, 2194-2203.

Oldfield, R. C. (1971). The assessment and analysis of handedness: The Edinburgh inventory. Neuropsychologia, 9, 97-113.

Oostenveld, R., Fries, P., Maris, E., \& Schoffelen, J.-M. (2011). FieldTrip: Open source software for advanced analysis of MEG, EEG, and invasive electrophysiological data. Computational Intelligence and Neuroscience, 2011, 9. http://dx.doi.org/10.1155/2011/156869

Orgs, G., Dombrowski, J.-H., Heil, M., \& Jansen-Osman, P. (2008). Expertise in dance modulates alpha/beta event-related desynchronization during action observation. European Journal of Neuroscience, 27, 33803384.

Patel, S. H., \& Azzam, P. N. (2005). Characterization of N200 and P300: Selected studies of the event related potential. International Journal of Medical Sciences, 2, 147-154.

Perry, A., \& Bentin, S. (2009). Mirror activity in the human brain while observing hand movements: A comparison between EEG desynchronization in the mu-range and previous fMRI results. Brain Research, 1282, 126-132.

Perry, A., Troje, N. F., \& Bentin, S. (2010). Exploring motor system contributions to the perception of social information: Evidence from EEG activity in the mu/alpha frequency range. Social Neuroscience, 5, 272-284.

Pfurtscheller, G., \& Aranibar, A. (1979). Evaluation of event-related desynchronization (ERD) preceding and following voluntary self-paced movements. Electroencephalography and Clinical Neurophysiology, 46, 138-146.

Pfurtscheller, G., \& Berghold, A. (1989). Patterns of cortical activation during planning of voluntary movement. Electroencephalography and Clinical Neurophysiology, 72, 250-258.

Pfurtscheller, G., \& Lopes da Silva, F. H. (1999). Event-related desynchronization and related oscillatory phenomena of the brain. Handbook of electroencephalography and clinical neurophysiology, Vol. 6, Amderdam: Elsevier.

Polich, J. (2007). Updating P300: An integrative theory of P3a and P3b. Clinical Neurophysiology, 118, 2128-2148. 
Reeves, B., Thorson, E., Rothschild, M. L., McDonald, D., Hirsch, J., \& Goldstein, R. (1985). Attention to television: Intrastimulus effects of movement and scene change on alpha variation over time. International Journal of Neuroscience, 27, 241-255.

Ruchsow, M., Spitzer, M., Grön, G., Grothe, J., \& Kiefer, M. (2005). Error processing and impulsiveness in normals: Evidence from even-related potentials. Cognitive Brain Research, 24, 317-325.

Salt, B. (2006). Moving into pictures. London: Starword. 2006.

Sbriscia-Fioretti, B., Berchio, C., Freedberg, D., Gallese, V., \& Umilta, M. A. (2013). ERP modulation during observation of abstract paintings by Franz Kline. PLoS ONE, 8(10), e75241. doi:10.1371/ journal.pone.0075241

Schröder, J. (1990). Die psychologische realität von prinzipien des continuity cinema. In G. Schumm \& H. G. Wulff (Eds.), Film und psychologie I. kognition-rezeption-perzeption (pp. 109-142). Münster, Germany: Maks Publikationen.

Schwan, S., \& Ildirar, S. (2010). Watching film for the first time: How adult viewers interpret perceptual discontinuities in film. Psychological Science, 21, 1-7.

Shmuelof, L., \& Zohary, E. (2005). Dissociation between central and dorsal fMRI activation during object and action recognition. Neuron, 47, 457-470.

Smith, T. (2006) An Attentional Theory of Continuity Editing. Free web version: http://hdl.handle.net/1842/ 1076

Smith, T. (2012). The attentional theory of cinematic continuity. Projections: The Journal for Movies and the Mind, 6(1), 1-27.

Stancak, A., \& Pfurtscheller, G. (1996). Mu-rhythm changes in brisk an slow self-paced finger movements. NeuroReport, 7, 1161-1164.

Steinbeis, N., \& Koelsch, S. (2008). Shared neural resources between music and language indicate semantic processing of musical tension-resolution patterns. Cerebral Cortex, 18, 1169-1178.

Uithol, S., van Rooij, I., Bekkering, H., \& Haselager, W. F. G. (2011). Understanding motor resonance. Social Neuroscience, 6(4), 388-397.

d'Ydewalle, G., \& Vanderbeeken, M. (1990). Perceptual and cognitive processing of editing rules in film. In R. Groner, G. d'Ydewalle, \& R. Parnham (Eds.), From eye to mind: Information acquisition in perception, search, and reading (pp. 129-139). Amsterdam: Elsevier (North Holland).

\section{Appendix}

This Appendix contains figures and results that, for better readability, where excluded from the main text.

\section{Additional figures: ERP analysis}

As explained in the main text, ERPs were computed by averaging over trials and participants using time-locked-analysis over the segmented epochs as implemented in Fieldtrip. Regions of interest were selected on the basis of the findings of prior studies investigating ERPs due to content/structure violations (Koelsch, 2011; Kutas \& Hillyard, 1980; Kutas \& Federmeier, 2000; Lau et al., 2008; Maffongelli et al., 2015; Steinbeis \& Koelsch, 2008). In these studies, usually four regions of interest (ROIs) were defined: anterior left, anterior right, posterior left, and posterior right. We adopted these ROIs and defined two further central ROIs to allow a comparison with results of the ERD analysis when considering differences between the two Montage conditions. The resulting six ROIs are indicated in Fig. A1. 


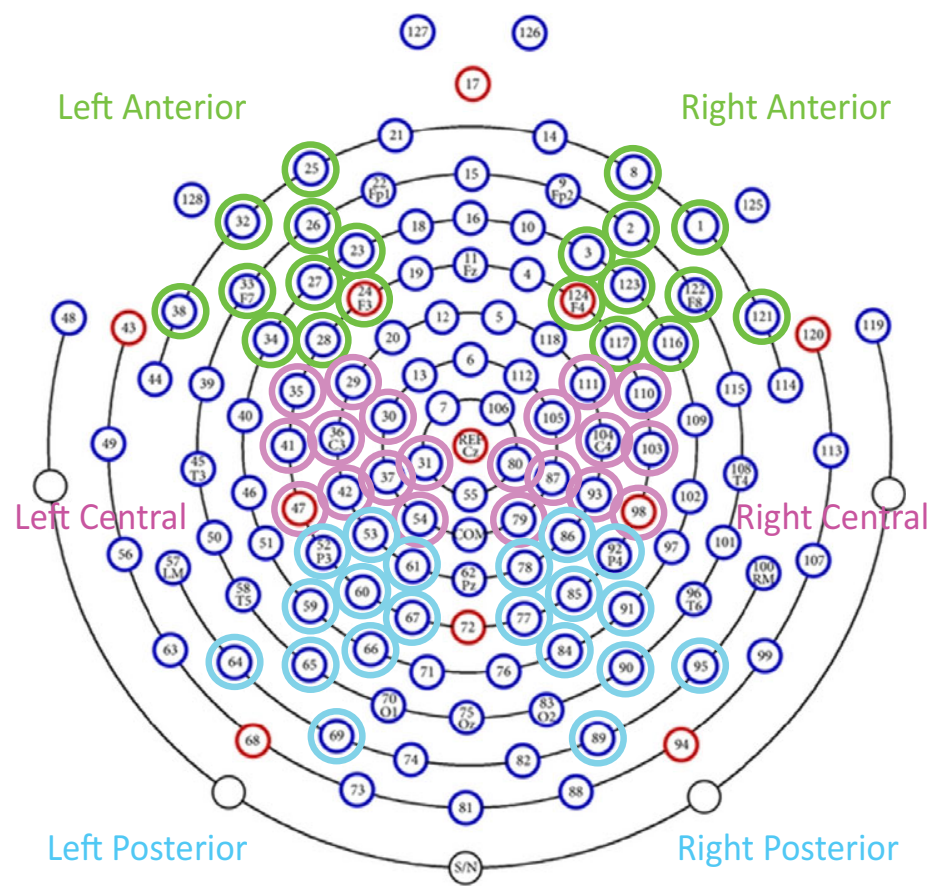

Fig. A1. Selection of electrodes for the six ROIs chosen.

Furthermore, time windows of interest were detected based on where (on scalp surface) ERPs reached their maximum values. As some components were rather near in timing, we especially in the early periods after point zero, chose quite narrow windows to not confound findings. This resulted in the following windows (see also Fig. A2):

Time window $1=140-190 \mathrm{~ms}$ after stimulus onset - centered on an early negativity (N1) in anterior and central regions and an early positivity (P1) in posterior regions.

Time window $2=180-220 \mathrm{~ms}$ after stimulus onset - centered on an early positivity in anterior and central regions (P2) and an early negativity in posterior regions (N2).

Time window $3=250-380 \mathrm{~ms}$ after stimulus onset - centered on a strong negativity in anterior and central regions (N3) and a strong positivity in posterior regions (P3).

Time window $4=400-650 \mathrm{~ms}$ after stimulus onset - centered on a late positivity (P46) in all regions.

\section{Additional figures: ERD analysis}

As explained in the main text electrode-clusters of interest were chosen for each of the two frequency bands of interest (8-14 and $15-24 \mathrm{~Hz}$ ) by means of a first exploratory statistical analysis comparing baseline and action execution condition in a cluster-based permutation test as implemented in FieldTrip (using dependent samples $t$-test statistics, 

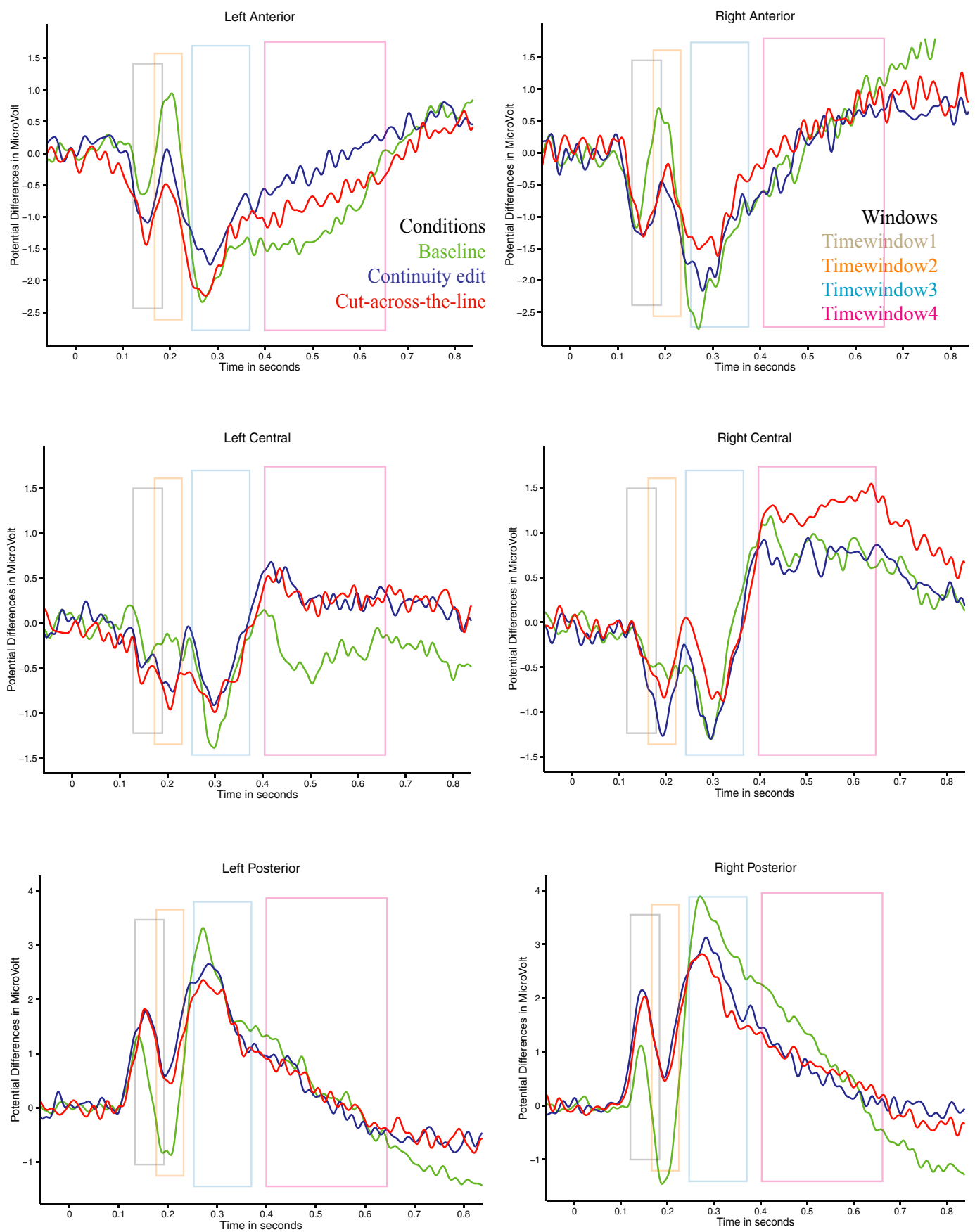

Fig. A2. ERP time course over the six selected ROI frames, marked by frames the four time windows chosen. 
Plotted results of cluster based permutation analysis comparing baseline and action execution condition (average of $100 \mathrm{~ms}$ before and after button press). Chosen electrode clusters for further analysis marked by white dashed line.

1) alpha $(8-14 \mathrm{~Hz})$

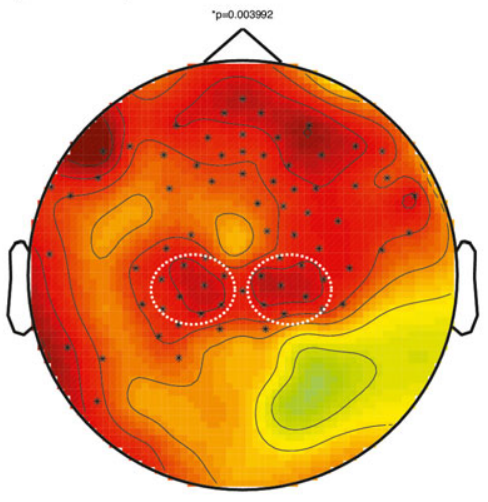

left central cluster: E 30, 31, 36, 37, 41, 42, 53, 54

right central cluster: E 79, 80, 86, 87, 93, 103, 104, 105
2) lower/middle beta $(15-24 \mathrm{~Hz})$

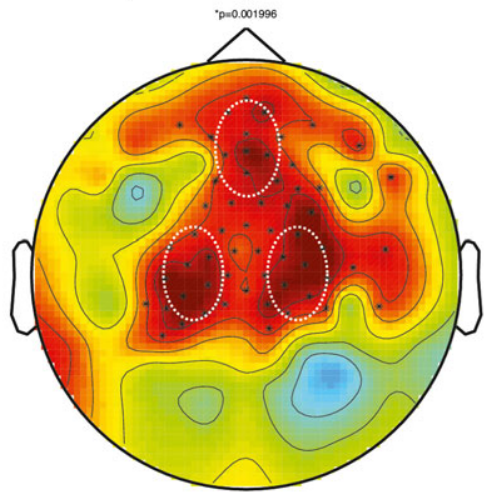

left central cluster: E $30,35,36,37,41,42,47,52,53$ right central cluster: E 86, 87, 93, 98, 103, 104, 105, 110, 111 frontal cluster: E 4, 10, 11, 14, 15, 16, 17, 19, 21,

Fig. A3. Electrode clusters chosen for the two band ranges of interest on the base of dependent samples $t$-test statistics using Monte Carlo Method.

Monte Carlo method, based on 500 randomizations). On the basis of the results showing the electrodes with significant differences (see topoplots below), for alpha as well as for beta bands, two symmetrical central clusters (one in the left, the other in the right hemisphere) were chosen. As previous research has described the source of the beta component of the mu-rhythm sometimes lying more frontal (see also Stancak \& Pfurtscheller, 1996), for beta bands, we additionally selected one frontal cluster also showing a strong difference between conditions; see Fig. A3.

\section{Complete ERD results}

For better readability in the main text, we did report in detail only those results related to our hypothesis. Below the complete results are provided.

A $4 \times 2$ ANOva (Condition $\times$ Hemisphere) comparing alpha-power in the selected clusters across conditions showed a significant main effect of Condition ( $F(3$, $48)=20.67, p<.001)$. Post hoc comparisons showed that frequency power for baseline was significantly higher than for all other conditions $(p<.001)$. Furthermore, frequency power for both observation conditions was significantly higher than for action execution (for continuity edit versus action execution $p<.01$, for cuts-across-the-line vs. action execution $p<.05$ ) (baseline MS: 0.262, STE: 0.21; continuity edit MS: -0.176 , STE: 0.214; cuts-across-the-line MS: -0.206 , STE: 0.22 , action execution MS: -0.446 , STE: 0.17); see Fig. 7. 
A $4 \times 3$ ANOVA (Condition $\times$ Region) comparing beta-power in the selected clusters across conditions accordingly showed a significant main effect for Condition ( $F(3$, $48)=25.63, p<.001)$, for Region $(F(2,32)=16.66, p<.001)$ and a significant Interaction Condition $\times$ Region $(F(6,96)=3.6, p<.01)$. Post hoc tests revealed that the significant differences in conditions again occurred between baseline and all other conditions (all $p<.01$ ) (baseline MS: -0.776, STE: 0.124, continuity edit MS: -1.159 , STE: 0.109, cuts-across-the-line: -1.16 , STE: 0.114, action execution: MS: -1.236 , STE: 0.095), for Regions between the two central clusters and the frontal cluster (all $p<.001$ ) (central left MS: -1.248 , STE: 0.123, central right MS: - 1.218, STE: 0.127, frontal MS: -0.782 , STE: 0.1). Post hoc tests for the Condition $\times$ Region Interaction showed that for left central electrodes as well as for frontal electrodes baseline beta power was significantly higher than the respective values for the observation conditions and for action execution $(p<.001)$. For right central electrodes also a difference between beta power in observation conditions and action execution was found $(p<.01$, for central left electrodes this difference was not significant $(p=0.55)$ ) (baseline left central MS: -0.983 , STE: 0.142, continuity edit left central MS - 1.309, STE: 0.132, cuts-across-the-line left central MS: -1.307, STE: 0.134, action execution left central MS: -1.392 , STE: 0.11, baseline right central MS: -0.95 , STE: 0.144, continuity edit right central MS: - 1.256, STE: 0.131, cuts-across-the-line right central MS: -1.264, STE: 0.133, action execution right central MS: -1.392, STE: 0.11, baseline frontal MS: -0.394, STE: 0.14, continuity edit frontal MS: -0.912, STE: 0.098, cuts-across-the-line frontal MS: -0.908, STE: 0.105, action execution frontal MS: -0.913, STE: 0.094); see Fig. 6.

For central alpha power values, the $2 \times 2 \times 5$ ANOvA ( 2 levels of Condition [continuity edit and cuts-across-the-line], 2 levels of Hemisphere [left vs. right], and 5 levels of Time [5 s from cut on]) showed a significant main effect of Time $(F(4,64)=26.10$, $p<.001)$ as well as a significant interaction Condition $\times \operatorname{Hemisphere}(F(1,16)=6.22$, $p<.05)$.

Post hoc comparisons showed that for the time course, the significant differences occurred between all time windows during video observation (desynchronization phase) and all time windows after video observation (resynchronization phase) $(p<.01)(1 \mathrm{st}$ second MS: -0.445, STE: 0.09, 2nd second MS: -0.449, STE: 0.11, 3rd second MS: -0.427 , STE: 0.08 , 4th second MS: -0.22, STE: 0.07, 5th second MS: -0.086 , STE: 0.07). See alpha and beta results in Fig. 8. Post hoc comparisons further investigating the significant Condition $\times$ Hemisphere Interaction showed that the significant differences occurred between left and right hemisphere for continuity edit conditions (with the left hemisphere being more strongly desynchronized than the right one, $p<.001$ ) and between continuity edits and cuts-across-the-line in the right hemisphere (with continuity edits being less desynchronized than cuts-across-the-line $p<.01$ ) (continuity edits left hemisphere MS: -0.3 , STE: 0.082 , continuity edits right hemisphere MS: -0.273 , STE: 0.079, cuts-across-the-line left hemisphere MS: -0.289, STE: 0.08, cuts-across-the-line right hemisphere MS: 0.291, STE: 0.078); see Fig. 7.

For central beta power values, the $2 \times 2 \times 5$ ANOvA ( 2 levels of Condition (continuity edit and cuts-across-the-line), 2 levels of Hemisphere (left vs. right), and 5 levels of Time 
$(5 \mathrm{~s}$ from cut on $)$ showed a significant main effect of Time only $(F(4,64)=23.53$, $p<.001)$.

Post hoc analyses showed that for the time course, the significant differences occurred between all time windows during video observation (desynchronization phase) and all time windows after video observation (resynchronization phase) $(p<.001)(1 \mathrm{st}$ second MS: -0.323 , STE: 0.074, 2nd second MS: -0.317 , STE: 0.075, 3rd second MS: -0.251 , STE: 0.064 , 4th second MS: -0.053 , STE: 0.065 , 5th second MS: -0.042 , STE: 0.058); see Fig. 8.

For frontal beta power values, the $2 \times 5$ ANOva ( 2 levels of Condition [continuity edits and cuts-across-the-line] and 5 levels of Time [ $5 \mathrm{~s}$ from cut on]) showed a significant main effect of Time only $(F(4,64)=17.36, p<.001)$.

Post hoc analyses showed that for the time course, the significant differences occurred between all time windows during video observation (desynchronization phase) and all time windows after video observation (resynchronization phase) (all $p<.001$ ) (1st second MS: -0.502 , STE: 0.093 , 2nd second MS: -0.516 , STE: 0.096, 3rd second MS: -0.47 , STE: 0.097 , 4th second MS: -0.362 , STE: 0.099, 5th second MS: -0.301, STE: 0.102); see Fig. 8.

To control for similar effects in occipital regions, we repeated the analysis just described in 1) and 2) in occipital electrodes. The $4 \times 2$ ANOVA with 4 factors of Condition (baseline, continuity edits, cuts-across-the-line and action execution) and 2 factors of Hemisphere in the occipital region showed a main effect of Condition only $(F(3,48)=21.44, p<.001)$. Post hoc comparisons showed that frequency power for baseline was significantly higher than for all other conditions $(p<.001)$ (baseline MS: 0.501 , STE: 0.224 , continuity edit MS: -0.104 , STE: 0.186 , cuts-across-the-line MS: -0.118 , STE: 0.185 , action execution MS: 0.017, STE: 0.194). There was no difference between frequency power for observation conditions and action execution $(p<.05)$.

The $2 \times 2 \times 5$ AnOva ( 2 levels of Condition [continuity edit and cuts-across-the-line], 2 levels of Hemisphere [left vs. right], and 5 levels of Time [ $5 \mathrm{~s}$ from cut on]), investigating differences between the two observation conditions showed a significant main effect of Time only $(F(4,64)=23.93, p<.001)$. Post hoc tests showed that for the time course, the significant differences occurred between all time windows during video observation (desynchronization phase) and all time windows after video observation (resynchronization phase) (all $p<.05$ ) (1st second MS: -0.565 , STE: 0.11 , 2nd second MS: -0.612 , STE: 0.12 , 3rd second MS: -0.586 , STE: 0.102, 4th second MS: -0.333 , STE: 0.102 , 5th second MS: -0.152 , STE: 0.146; see Fig. 9.

\section{Rating task}

In the main text, we only reported the significant results of the rating task.

Results of the other questions were as follows:

Question 1 ("How involved did you feel in the scene?") Continuity edit MS: 25.3, STE: 3.39, Cut-across-the-line MS: 25.09, STE: 3.74 
Question 2 ("How much did you feel like the actor [the one handing over the object]?") Continuity edit MS: 31.6, STE: 4., Cut-across-the-line MS: 36.89, STE: 6.06

Question 3 ("How easy to watch did you find this video?") Continuity edit MS: 61.6, STE: 6.29, Cut-across-the-line MS: 62.26, STE: 6.63

Question 5 ("Which of the two videos was longer?"-Mean corresponds to the amount of videos of that type rated longer) Continuity edit MS: 7.76, STE: 0.63 Cut-across-theline MS: 8.24, STE: 0.63 